Document downloaded from:

http://hdl.handle.net/10251/50721

This paper must be cited as:

Fernández Diego, M.; González-Ladrón-De-Guevara, F. (2014). Potential and limitations of the ISBSG dataset in enhancing software engineering research: A mapping review. Information and Software Technology. 56(6):527-544. doi:10.1016/j.infsof.2014.01.003.

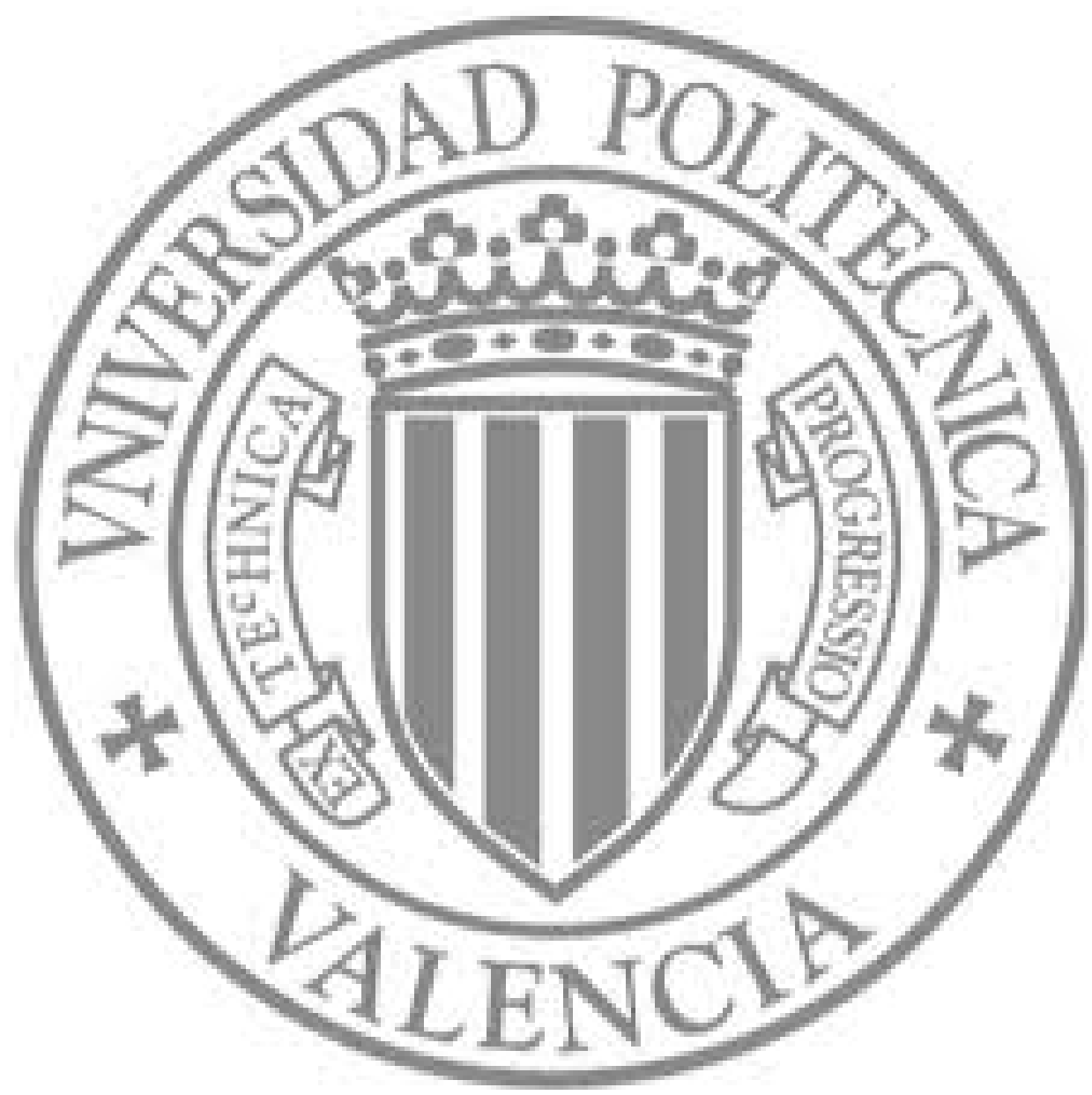

The final publication is available at

http://dx.doi.org/10.1016/j.infsof.2014.01.003

Copyright Elsevier 


\title{
Potential and Limitations of the ISBSG Dataset in Enhancing Software Engineering Research: A Mapping Review
}

\author{
Marta Fernández-Diego*, Fernando González-Ladrón-de-Guevara \\ Business and Economics Department, \\ Universitat Politècnica de València, \\ Camino de Vera, s/n, 46022 Valencia, Spain.
}

\author{
*Corresponding author. Tel.: +34 9638776 85. Fax: +34 963879779 \\ E-mail address: marferdi@omp.upv.es (Marta Fernández-Diego), fgonzal@omp.upv.es (Fernando \\ González-Ladrón-de-Guevara)
}

\section{Abstract}

Context: The International Software Benchmarking Standards Group (ISBSG) maintains a software development repository with over 6,000 software projects. This dataset makes it possible to estimate a project's size, effort, duration, and cost.

Objective: The aim of this study was to determine how and to what extent, ISBSG has been used by researchers from 2000, when the first papers were published, until June of 2012.

Method: A systematic mapping review was used as the research method, which was applied to over 129 papers obtained after the filtering process.

Results: The papers were published in 19 journals and 40 conferences. Thirty-five percent of the papers published between years 2000 and 2011 have received at least one citation in journals and only five papers have received six or more citations. Effort variable is the focus of $70.5 \%$ of the papers, $22.5 \%$ center their research in a variable different from effort and $7 \%$ do not consider any target variable. Additionally, in as many as $70.5 \%$ of papers, effort estimation is the research topic, followed by dataset properties (36.4\%). The more frequent methods are Regression (61.2\%), Machine Learning (35.7\%), and Estimation by Analogy (22.5\%). ISBSG is used as the only support in $55 \%$ of the papers while the remaining papers use complementary datasets. The ISBSG release 10 is used most frequently with 32 references. Finally, some benefits and drawbacks of the usage of ISBSG have been highlighted.

Conclusion: This work presents a snapshot of the existing usage of ISBSG in software development research. ISBSG offers a wealth of information regarding practices from a wide range of organizations, applications, and development types, which constitutes its main potential. However, a data preparation process is required before any analysis. Lastly, the potential of ISBSG to develop new research is also outlined.

\section{Keywords}

Systematic mapping study

Research methods

Software engineering

ISBSG 
Software effort estimation

Software cost prediction

\section{Introduction}

The International Software Benchmarking Standards Group [1] designed and currently maintains two international public repositories: Software Development \& Enhancement with over 6,000 software projects and Maintenance \& Support with over 470 software applications. The repository contains more than 150 data fields collected from a wide range of countries, organizations, application types, and development types.

The goal of ISBSG is to help any type of organization (business, public, or non-profit) improve their IT resource management through the use of these datasets by performing their own analyses, estimations, comparisons, or benchmarking.

The ISBSG dataset for software development is organized by releases. The current version (since 2013) is release 12, which includes over 6,000 projects distributed between years 1989 and 2013. Such a dataset makes it possible to estimate a project's size, effort, duration, and cost. Furthermore, ISBSG enables users to check project specification completeness, reduce project risk, control software development, plan infrastructure development, and benchmark performance. But ISBSG suffers from two major problems: the heterogeneity of data, i.e., the combining of data from heterogeneous sources [2], and most of the variables have a lot of missing values.

ISBSG implements two fields in its datasets, which can be used to filter out low quality cases from the analysis and help handle data validation and rating issues [S105]. Each project submitted to the ISBSG repository is validated against specific quality criteria and rated in four categories. As pointed out in Liebchen and Shepperd [3], the classification is primarily guided by the completeness of the software projects, which means that low quality data are interpreted as possessing high levels of missing values.

A mapping study can be considered as a secondary study that reviews articles related to a specific research topic. Such a study provides an overview of a research area to assess the existing evidence [4] and can identify gaps in the set of primary studies, where new or better primary studies are required. Mapping studies also pinpoint specific realms of knowledge where there may be an opportunity for more complete systematic literature reviews to be undertaken $[5,6]$.

Ultimately, a mapping study aims at providing a classification, conducting a thematic analysis, or presenting publication channels [7]. Petticrew and Roberts [5] also suggest that this type of study "involves a search of the literature to determine what sorts of studies addressing the systematic review question have been carried out, where they are published, in what databases they have been indexed, what sorts of outcomes they have assessed, and in which populations." These studies require a rigorous searching process as well as detailed inclusion and exclusion criteria that are clearly defined in the research 
protocol and presented in the results report [8]. The main difference between a mapping study and a systematic literature review is the formulation of the research questions and the analysis of the available information [6].

The purpose of this study was to determine to what extent and how ISBSG has been the support for a group of researchers in software engineering. Thereby, systematic mapping has been performed to "map out" papers that have used this dataset in an attempt to identify the topics, estimation methods, complementary datasets, and other issues that have been dealt with in research questions. In this way, a picture portraying the potential and limitations of ISBSG as a research facility has been obtained. Additionally, the investigation into the set of papers related to ISBSG and their classification are valuable results [9].

The rest of this paper will be organized as follows: Section 2 will describe the mapping process, Section 3 will report the mapping results, Section 4 will discuss the limitations of the study, and finally, Section 5 will outline the main conclusions obtained and future work lines.

\section{Research methodology}

Systematic mapping studies are a type of systematic literature review that aims to collect and classify research papers related to a specific topic [5-7,10,11].

This section provides an overview of the steps involved in the process of mapping review following Petersen et al. [7] including the formulation of the research questions, the search strategy for primary studies, the inclusion and exclusion criteria, and the data collection process.

\subsection{Research questions}

The primary goal addressed by this study is to analyze the use of the ISBSG dataset by the research community and to consider its potential to undertake the appropriate scientific research. A number of issues and their rationales were considered:

- Research question number 1 (RQ1): Which and how many journals and conferences include ISBSG-based research papers? The main purpose of this question is to provide a list of journals and conferences interested in such studies and to assess the relevance of papers based on ISBSG. To acknowledge the prestige associated with each journal and conference, their rankings are discussed. Furthermore, the temporal evolution of paper publications based on ISBSG as primary data was also taken into account for the most relevant journals and conferences.

- RQ2: To what extent are researchers working with the ISBSG dataset aware of other works also using the ISBSG dataset? By examining the citations between papers, it is possible to assess the awareness of researchers to the ISBSG body of 
knowledge and their ability to contribute to this body of knowledge. By scrutinizing the citations, it is possible to analyze the evolution of received citations over time.

- RQ3: What are the most investigated research topics and their trends over time? The motivation behind this question is to provide an update on the contents of ISBSG-related research. Furthermore, by analyzing the evolution of the topics, both emerging issues and obsolete issues can be identified.

- RQ4: What are the effort estimation methods and what are their trends over time? The goal here is to provide insight as to which effort estimation methods (regression, machine learning methods, estimation by analogy, etc.) have been used in identified references and to determine their usage intensity over a period of time.

- RQ5: Is ISBSG data a valuable source of data for the research undertaken in the paper? This question can be answered by examining whether and why ISBSG is used alone or in combination with other datasets. These other datasets have been identified through a detailed analysis. Additionally, the ISBSG release number used by the researchers was identified. When compiled, this information can provide an indication of what advantges and limitations may exist when the ISBSG dataset is used.

\subsection{Search for primary studies strategy}

The following four bibliographic databases were used to make a general search for relevant papers in journals and conference proceedings: IEEE Xplore (all IEEE online publications), ACM Digital Library (ACM journals, newsletter articles, and conference proceedings), ScienceDirect (Elsevier Reference Works), and Web of Knowledge. These databases were selected because they are the major search engines and digital libraries most frequently used in systematic literature reviews performed by the software engineering community [12].

The search term "ISBSG" was used as the input for these search engines. This resulted in $134,63,57$, and 52 results respectively. Since only conference papers and journal articles were considered, three items from ACM and three from SD were eliminated. Following this elimination, 300 references were obtained before identifying and deleting duplicate records. The search was completed in June 2012.

Next, duplicate articles in these searches were eliminated. There were 37 duplicate articles and one article in triplicate from the four databases. In the end, 261 references remained. The overall primary study selection process is summarized in Fig. 1. 


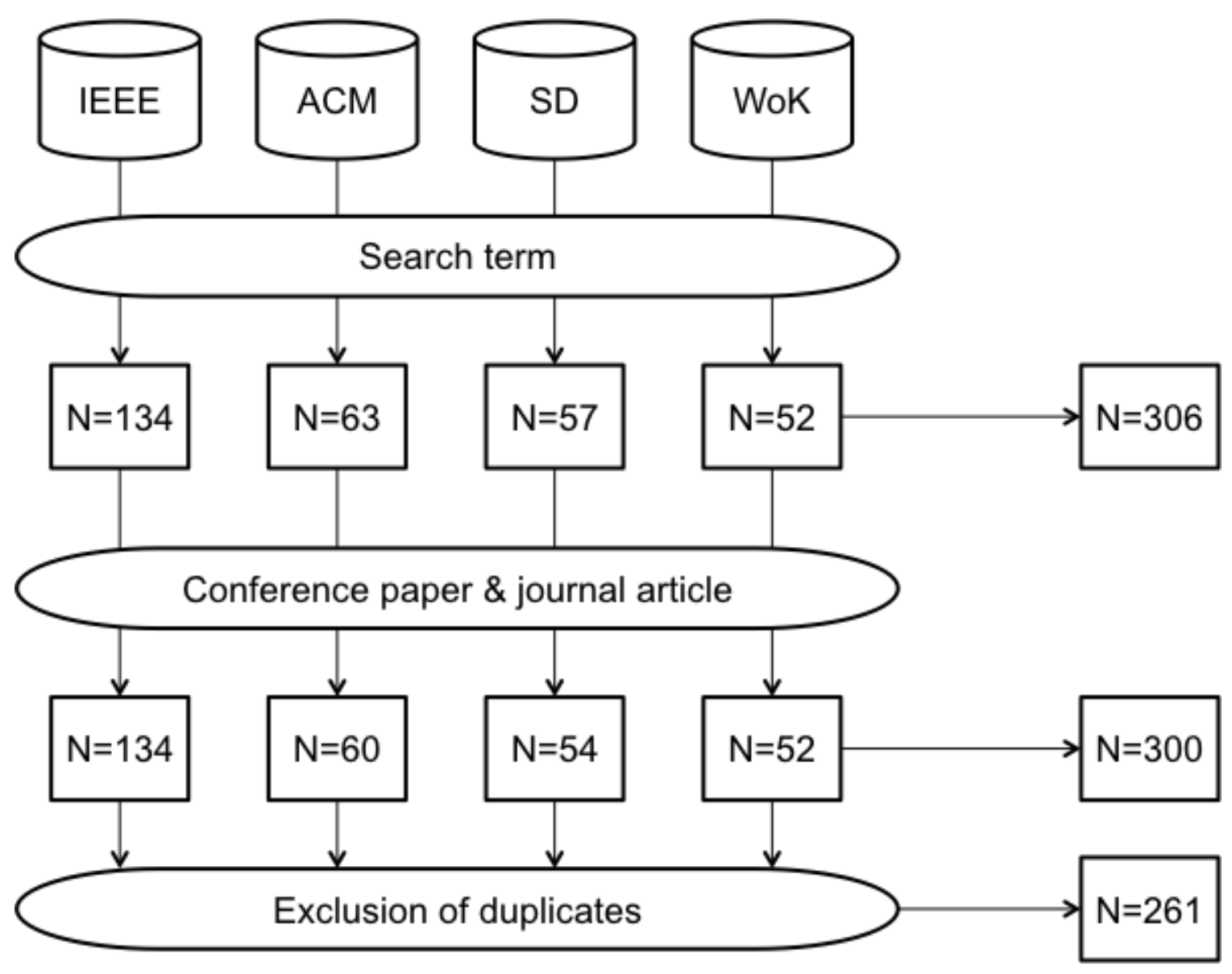

Figure 1: Search process for the selection of studies.

To make the search as inclusive as possible, no limits were placed on the date of publication and the number of restrictions was minimized. Therefore, no logical operators were used and the unique search term ('ISBSG') was applied not only to the title and abstract of the paper, but also to the body of the text. The search, however, within the full document record was not possible in papers indexed in the Web of Knowledge.

Consequently, an additional search was performed within the journals only indexed in the Web of Knowledge using their publisher's search engine. As a result, 21 additional papers were included and the set of analyzed papers increased to 282. These 21 additional papers were published in Empirical Software Engineering (10, Springer), the Software Quality Journal (6, Springer), the International Journal of Software Engineering and Knowledge Engineering (2, Word Scientific), and the Journal of Software Maintenance and Evolution-Research and Practice (3, Wiley).

\subsection{Inclusion and exclusion criteria}

Inclusion and exclusion criteria are required to assess each potential primary study. During the process, two false records were detected from the set of papers and two more were excluded since they were not written in English, resulting in 278 useable primary studies. 
In order to improve its reliability, the filtering process based on inclusion and exclusion criteria was performed by the two authors who completed the research explained in this publication. All conflicts were resolved via discussion. How the filtering procedure was performed on 278 items and resulted in a final subset of 129 papers is presented in Fig. 2. A cross-check of the selected articles has been carried out.

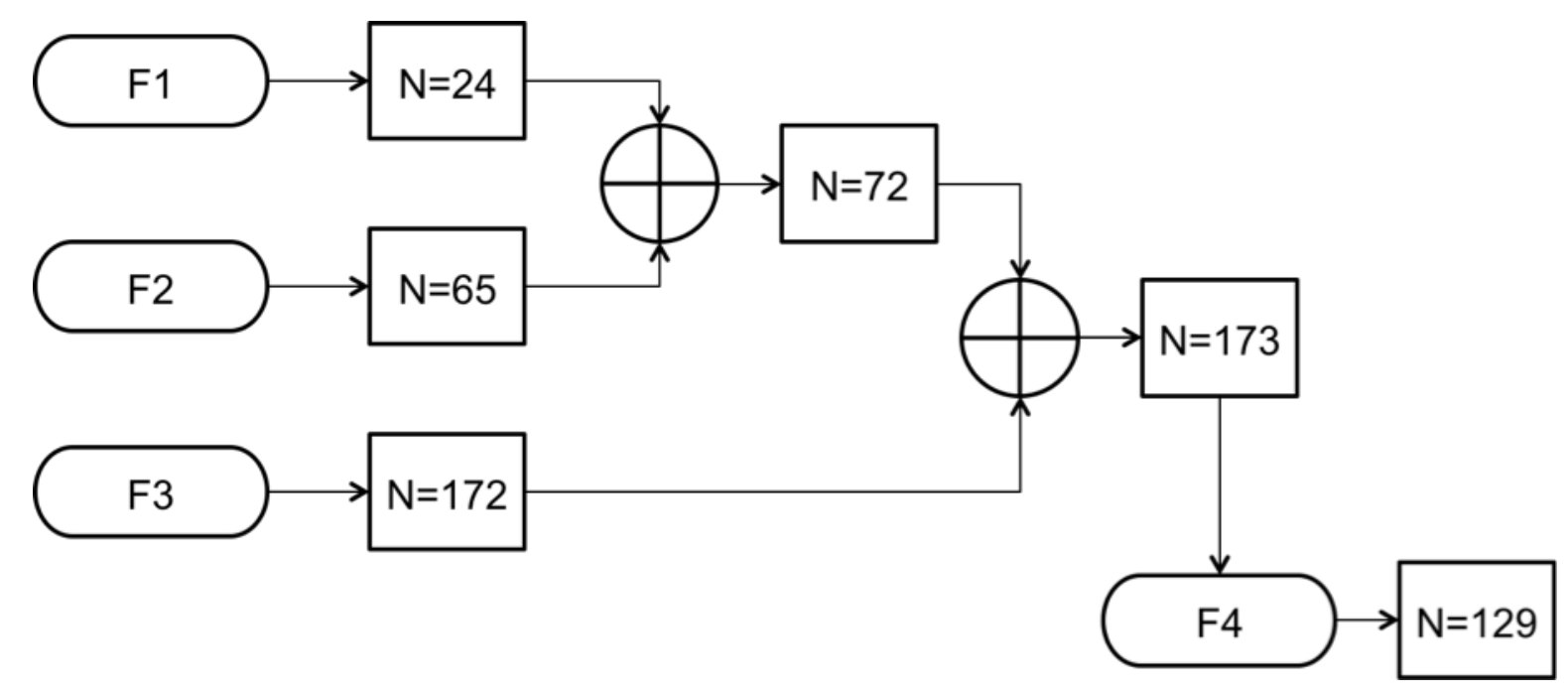

Figure 2: Filtering process.

In the first step, three filters were defined to verify that the references were related to the ISBSG dataset. The following list describes the filtering process:

- F1: The first filter (F1) was used to identify those references in which the term ISBSG appears in either the title or the keywords (24 references were detected with this condition).

- F2: The second filter (F2) was used to identify those papers in which the term 'ISBSG' appears in the abstract ( 65 references fulfilled this condition). The set of references that met the logical condition (F1 OR F2) included 72 references.

- F3: The third filter (F3) was used to locate the occurrences of the term ISBSG throughout the text. It was decided that a reference should be filtered out through F3 in the following situations:

- when 'ISBSG' only appeared in the list of references;

- when ISBSG was mentioned as an example of a dataset or when the publication simply referred to ISBSG;

- in cases where the ISBSG dataset was not used in the identified paper although it was mentioned that the authors of the paper intended to use ISBSG.

With the resulting 173 papers that satisfy the logical condition (F1 OR F2 OR F3), a second check was completed to verify whether data from the ISBSG dataset was used for the research undertaken in the paper. Articles where the ISBSG data was not the unique 
data source were still included. To summarize, whenever the ISBSG dataset is used in any way, the article passed the F3 filter. Since the use of ISBSG as a primary data source is of interest, an additional filter (F4) was created:

- F4: This final filter has been proposed to categorize how ISBSG was used: if ISBSG is used as a dataset, then $F 4=1$, otherwise $F 4=0$.

\subsection{Data collection}

After the filtering process, the most relevant information was obtained from each of the 129 remained studies. This includes both general information and data addressing the five research questions $[7,13]$. After reading each paper, the data was extracted and stored in a spreadsheet using the data extraction form presented in Table 1. Note that the first $R Q$ could be answered by processing just the general information collected for each paper.

Table 1: Data extraction form to collect information from each identified paper.

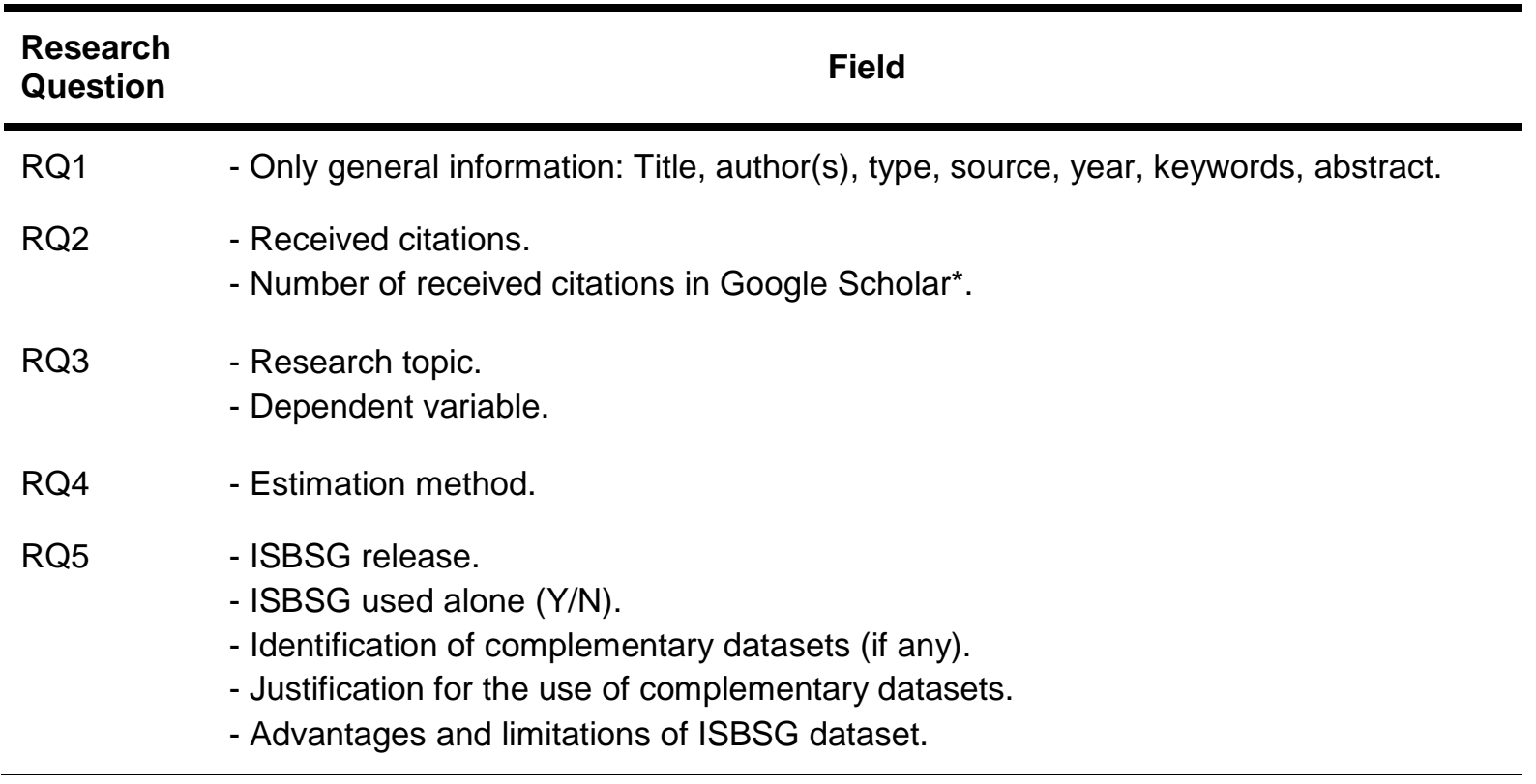

\footnotetext{
* Complementary information obtained from external sources.
}

The extraction form includes 17 fields for each paper. During data extraction, short notes were captured within the appropriate columns related to the topic, proposed methods, and complementary datasets. The description, the source, and whether they are public, limited, or private are examples of what was included in the notes. The goal of these notes was to capture as much information as possible in order to respond properly to the proposed research questions.

\section{Results}


The results of the systematic mapping study are presented following each of the research questions.

\subsection{RQ1}

Which and how many journals and conferences include ISBSG-based research papers?

Of the 129 remaining papers, 62 are journal articles and 67 are conference papers. As many as 19 journals with ISBSG-based research papers were found and are displayed in Table 2 with the corresponding number of identified papers per journal, proportion of identified papers compared to the total number of identified papers in the journals listed, and cumulative proportions of papers. The column titled "Ranking" in Table 2 shows the ranking of each journal in the category Computer Science, Software Engineering within the Journal Citation Report. If a journal does not belong to this category, the best quartile position in any category is shown followed by an asterisk.

Three journals, Journal of Systems and Software, Information and Software Technology, and Empirical Software Engineering, include $48.4 \%$ of all identified journal papers, with 11, 10 , and 9 papers respectively. They belong to the third, second, and first quartile correspondingly in the Computer Science, Software Engineering category. This category is the most common among the journals listed. All journals except SIGSOFT, which provides a forum for informal articles and other information about software engineering, are indexed within the Journal Citation Report.

Table 2: Journal type publication sources of selected papers.

\begin{tabular}{|c|c|c|c|c|}
\hline Journal & Number & Proportion \% & $\begin{array}{l}\text { Cumulative } \\
\text { proportion \% }\end{array}$ & nking \\
\hline Journal of Systems and Software & 11 & 17.7 & 17.7 & Q3 \\
\hline Information and Software Technology & 10 & 16.1 & 33.9 & Q2 \\
\hline Empirical Software Engineering & 9 & 14.5 & 48.4 & Q1 \\
\hline Software Quality Journal & 7 & 11.3 & 59.7 & Q4 \\
\hline \multicolumn{5}{|l|}{ International Journal of Software Engineering and } \\
\hline Knowledge Engineering & 3 & 4.8 & 64.5 & Q4 \\
\hline $\begin{array}{l}\text { Journal of Software Maintenance and Evolution } \\
\text { Research and Practice }\end{array}$ & 3 & 4.8 & 69.4 & Q3 \\
\hline Information \& Management & 2 & 3.2 & 72.6 & Q1* \\
\hline Journal of Computer Science and Technology & 2 & 3.2 & 75.8 & Q3 \\
\hline SIGSOFT Softw. Eng. Notes & 2 & 3.2 & 79.0 & \\
\hline
\end{tabular}




\begin{tabular}{lcccc} 
Software Engineering, IEEE Transactions on & 2 & 3.2 & 82.3 & Q1 \\
Software, IEEE & 2 & 3.2 & 85.5 & Q1 \\
Software, IET & 2 & 3.2 & 88.7 & Q4 \\
ACM Trans. Softw. Eng. Methodol. & 1 & 1.6 & 90.3 & Q2 \\
Annals of Operations Research & 1 & 1.6 & 91.9 & Q3* \\
Commun. ACM & 1 & 1.6 & 93.5 & Q1 \\
Computing and Informatics & 1 & 1.6 & 95.2 & Q4* \\
Expert Systems with Applications & 1 & 1.6 & 96.8 & Q1 \\
Information Processing Letters & 1 & 1.6 & 98.4 & Q4* \\
Science of Computer Programming & 1 & 1.6 & 100.0 & Q3 \\
\hline
\end{tabular}

* Quartile in category other than Computer Science, Software Engineering.

The 67 conference papers that were selected came from 40 different conferences. Table 3 shows the conferences with two or more ISBSG-based research papers (13), the number of papers, share, cumulative share and tier ranking in the CORE (Computing Research and Education Association of Australasia) conference list. These conferences include $59.7 \%$ of all identified conference papers. Between them, the most relevant conferences in terms of number of papers published related to the ISBSG dataset, are PROMISE (International Conference on Predictive Models in Software Engineering) and METRICS (International Software Metrics Symposium), which are not ranked by [14] administered by the Australian Research Council. They are followed by ESEM (International Symposium on Empirical Software Engineering and Measurement), which joined together ISESE and METRICS in 2007. ESEM and all other conferences with the exception of one are ranked in the CORE conference list. ESEM is the only conference in Table 3 with an " $A$ " ranking. The most common field for the listed conferences is Computer Software. If a conference does not belong to this field, the tier ranking in its field is shown followed by an asterisk. Note that one of the conferences appears with a single paper. The reason for this is because it is a joint conference of an international workshop and conference that does appear in the row above with two papers.

Table 3: Conference type sources with two or more selected papers.

\begin{tabular}{llccc}
\hline \multicolumn{1}{c}{ Conference } & Acronym & Number Proportion \% & $\begin{array}{c}\text { Cumulative } \\
\text { proportion \% Ranking }\end{array}$ \\
\hline $\begin{array}{llll}\text { International Conference on Predictive } \\
\text { Models in Software Engineering }\end{array}$ & PROMISE & 8 & 11.9 & 11.9
\end{tabular}


International Software Metrics

Symposium

METRICS

6

9.0

20.9

International Symposium on Empirical

Software Engineering and

Measurement

ESEM

4

6.0

26.9

A

Asia-Pacific Software Engineering

Conference

APSEC

3

4.5

31.3

C

International Conference on Computer

and Information Technology

CIT

3

4.5

35.8

$\mathrm{C}^{*}$

International Symposium on Empirical

Software Engineering

ISESE

3

4.5

40.3

B

International Computer Software and

Applications Conference

COMPSAC

2

3.0

43.3

B

Euromicro Conference on Software

Engineering and Advanced

Applications

SEAA

2

3.0

46.3

C

International Conference on Tools

with Artificial Intelligence

ICTAI

2

3.0

49.3

$B^{*}$

International Conference on Software

Process and Product Measurement

MENSURA

2

3.0

52.2

$\mathrm{C}^{*}$

Joint Conference of International

Workshop on Software Measurement and International Conference on

Software Process and Product

Measurement

IWSM-

Symposium on Applied Computing

MENSURA

1

1.5

53.7

$C^{*}$

SAC

2

3.0

56.7

$B^{*}$

World Academy of Science,

Engineering and Technology

2

3.0

59.7

* Tier ranking in field other than Computer Software. 


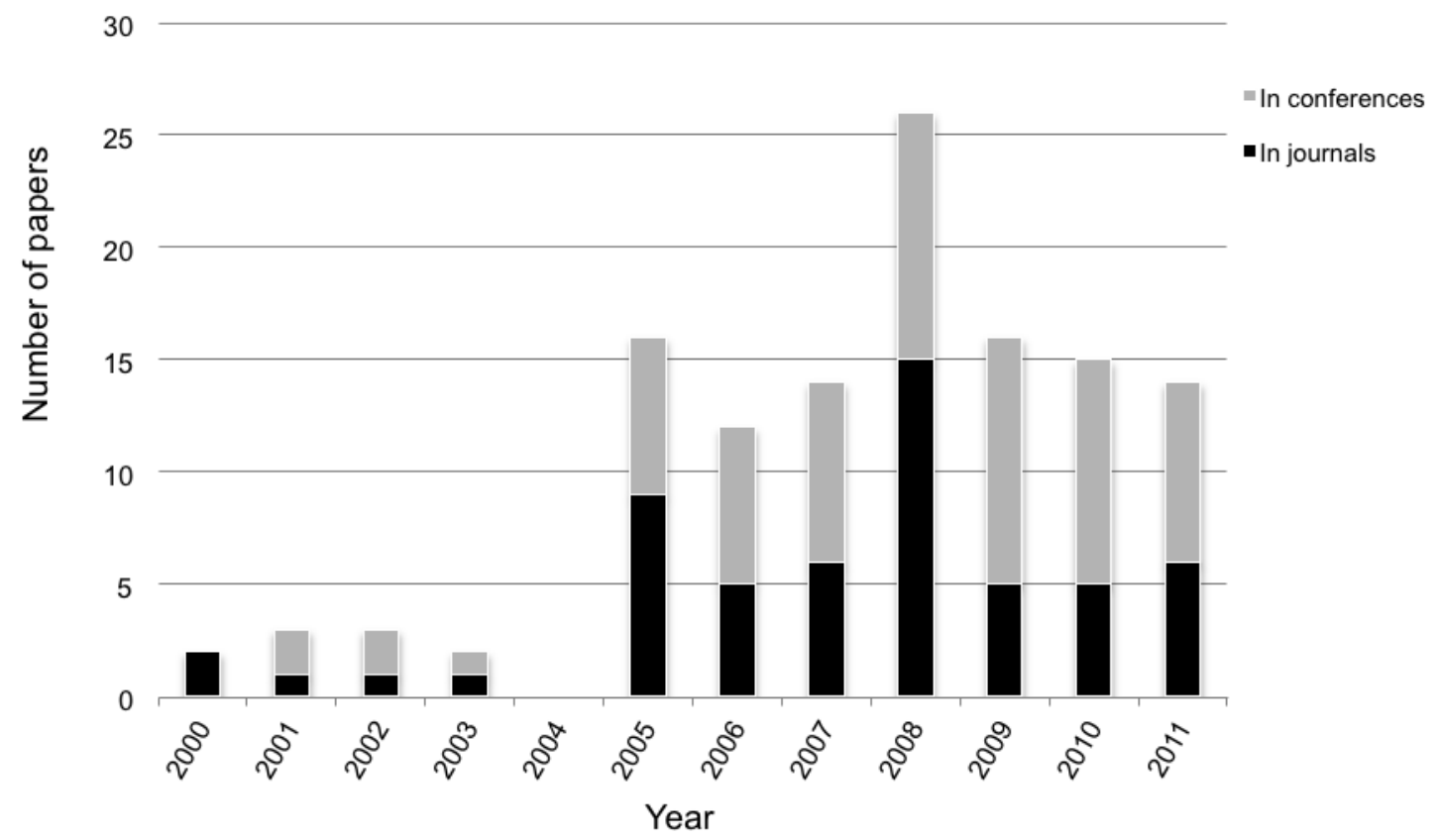

Figure 3: Number of papers in journals and conferences over time.

Fig. 3 shows that since an initial period with a slight acknowledgement of the ISBSG dataset and a full disappearance in 2004, there has been a period of time from 2005 to 2011 with a nearly constant presence of these types of research either in conferences or in journals (average $=16$ references) with a maximum in 2008 (26 references). The number of references published in conferences is usually greater than the number of papers published in journals.

A similar behavior can be observed in Fig. 4, which shows the progression of the number of papers published in Journal of Systems and Software, Information and Software Technology, and Empirical Software Engineering that together represent $48.4 \%$ of the identified papers. In 2000, two papers appeared in Information and Software Technology and again in 2005, after a gap of several years, two additional papers were published. A peak can be observed in 2008 with four papers. Since 2010, however, no more papers have been published. In the other two journals, a more uniform trend can be detected from 2005 to 2011 , with a peak also corresponding to year 2008. 


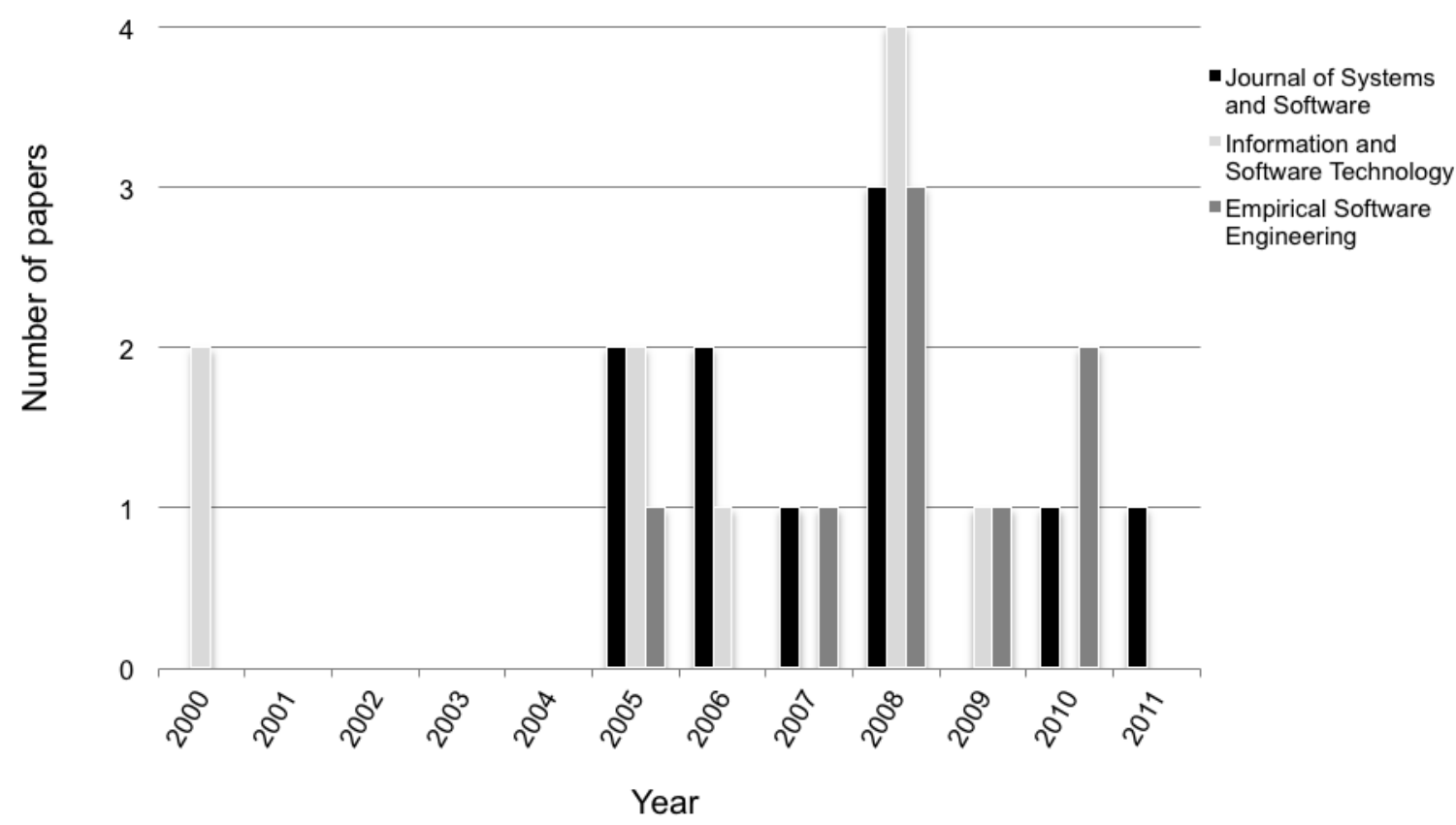

Figure 4: Number of papers published per year.

The distribution of conference papers over the years is more dispersed. Accordingly, PROMISE is a young conference, which began in 2005, that gives priority to empirical studies based on publicly available data repositories. PROMISE published three papers in 2008, one in 2010, and four in 2011. It currently holds 142 datasets, which are usually used to cross-check with several analyses. Moreover, METRICS published two papers in 2001 based on the ISBSG dataset and four in 2005 in its most recent edition.

\subsection{RQ2}

To what extent are researchers working with the ISBSG dataset aware of other works that also use the ISBSG dataset?

The best measure of awareness of research findings published in academic journals is the degree to which these journals or a specific paper within a journal are read. This information, however, is difficult to obtain and even some academics may obtain their information about specific research through secondary and tertiary distributors. Alternatively, the degree of awareness can be based on the number of times the identified papers have been cited by other papers [15]. Despite limitations, this indicator can provide some valuable information regarding the spread of scientific ideas over time.

Therefore, the number of citations that the 129 identified papers have received within the journal papers (62) of this same set have been considered. There are 44 identified papers (35.8\%) in the period from 2000 to 2011 that have received at least one citation in journals. Twenty-seven of these cited papers are journal papers. All in all, the identified papers have 
received 134 citations, which is an average of 1.04 citations per paper. Within these 44 cited papers, an average of 3.05 citations with a standard deviation of 3.5 have been received.

Within the 62 journal papers, the average number of citations is 33.2 with a standard deviation of 18.6 and a maximum value of 108 citations [S51]. The variable that describes the number of citations that have received the papers in the set by the papers also included in this set that have been published in journal papers (62) has a mean value of 2.2 with a standard deviation of 2.2 and a maximum value of 9 references that have been cited [S19]. Considering the ratio of citations that are received by the identified articles to the citations included in the journal papers, an average of $7.2 \%$ of citations used by journal papers are received by the articles identified in the set.

On the other hand, the list of papers that have received six or more citations from the journal papers subset is shown in Table 4. Additionally, Table 4 displays the source of the paper and the received citations delivered by Google Scholar.

Table 4: Top-cited identified papers with at least 6 citations.

\begin{tabular}{|c|c|c|c|c|}
\hline Ref. & Source & Year & $\begin{array}{c}\text { Number of received } \\
\text { citations }\end{array}$ & $\begin{array}{l}\text { Received citations } \\
\text { Google Scholar }\end{array}$ \\
\hline [S125] & METRICS & 2001 & 15 & 145 \\
\hline \multirow[t]{2}{*}{ [S40] } & METRICS & 2001 & 14 & 91 \\
\hline & $\begin{array}{l}\text { Information and } \\
\text { Software }\end{array}$ & & & \\
\hline [S4] & $\begin{array}{l}\text { Technology } \\
\text { Information and } \\
\text { Software }\end{array}$ & 2000 & 13 & 126 \\
\hline [S114] & $\begin{array}{l}\text { Technology } \\
\text { Information and } \\
\text { Software }\end{array}$ & 2005 & 10 & 78 \\
\hline [S23] & Technology & 2000 & 6 & 57 \\
\hline
\end{tabular}

Only five papers received six or more citations. The two conference papers came from METRICS and the three other papers were published in the Information and Software Technology journal. Since four out of five of the most cited papers corresponded to the beginning of the time period (2000-2001), it was decided that conducting a temporal analysis would be valuable. This is presented in Table 5 and Fig. 5.

Table 5: Citations received by identified papers in dataset journals over time. 


\begin{tabular}{|c|c|c|c|c|c|c|c|}
\hline Year & $\begin{array}{l}\text { Identified } \\
\text { articles }\end{array}$ & $\begin{array}{c}\text { Cited } \\
\text { articles }\end{array}$ & $\begin{array}{l}\text { Number } \\
\text { of } \\
\text { received } \\
\text { citations }\end{array}$ & $\begin{array}{l}\text { Identified } \\
\text { cited } \\
\text { articles } \\
\%\end{array}$ & $\begin{array}{l}\text { Received } \\
\text { citations } \\
\text { per } \\
\text { identified } \\
\text { article }\end{array}$ & $\begin{array}{l}\text { Received } \\
\text { citations } \\
\text { per cited } \\
\text { article }\end{array}$ & $\begin{array}{c}\text { Received } \\
\text { citations } \\
\text { on Google } \\
\text { Scholar }\end{array}$ \\
\hline 2000 & 2 & 2 & 19 & 100.0 & 9.5 & 9.5 & 183 \\
\hline 2001 & 3 & 3 & 34 & 100.0 & 11.3 & 11.3 & 275 \\
\hline 2002 & 3 & 1 & 1 & 33.3 & 0.3 & 1.0 & 38 \\
\hline 2003 & 2 & 1 & 3 & 50.0 & 1.5 & 3.0 & 37 \\
\hline 2004 & 0 & 0 & 0 & 0.0 & 0.0 & 0.0 & - \\
\hline 2005 & 16 & 9 & 26 & 56.2 & 1.6 & 2.9 & 222 \\
\hline 2006 & 12 & 7 & 19 & 58.3 & 1.6 & 2.7 & 175 \\
\hline 2007 & 14 & 5 & 9 & 35.7 & 0.6 & 1.8 & 150 \\
\hline 2008 & 26 & 11 & 18 & 42.3 & 0.7 & 1.6 & 176 \\
\hline 2009 & 16 & 3 & 3 & 18.7 & 0.2 & 1.0 & 55 \\
\hline 2010 & 15 & 2 & 2 & 13.3 & 0.1 & 1.0 & 26 \\
\hline 2011 & 14 & 0 & 0 & 0.0 & 0.0 & 0.0 & - \\
\hline Total & 123 & 44 & 134 & 35.8 & 1.1 & 3.0 & 1337 \\
\hline
\end{tabular}

Fig. 5 shows that during the initial period from 2000 to 2001, the five articles that were cited received 53 citations from papers within the set published in journals. This means an important share $(39.5 \%)$ of the sum of received citations, with an average of 10.6 citations per article. Therefore, it is apparent that the impact of these articles is greater than the impact for the remaining identified articles. 


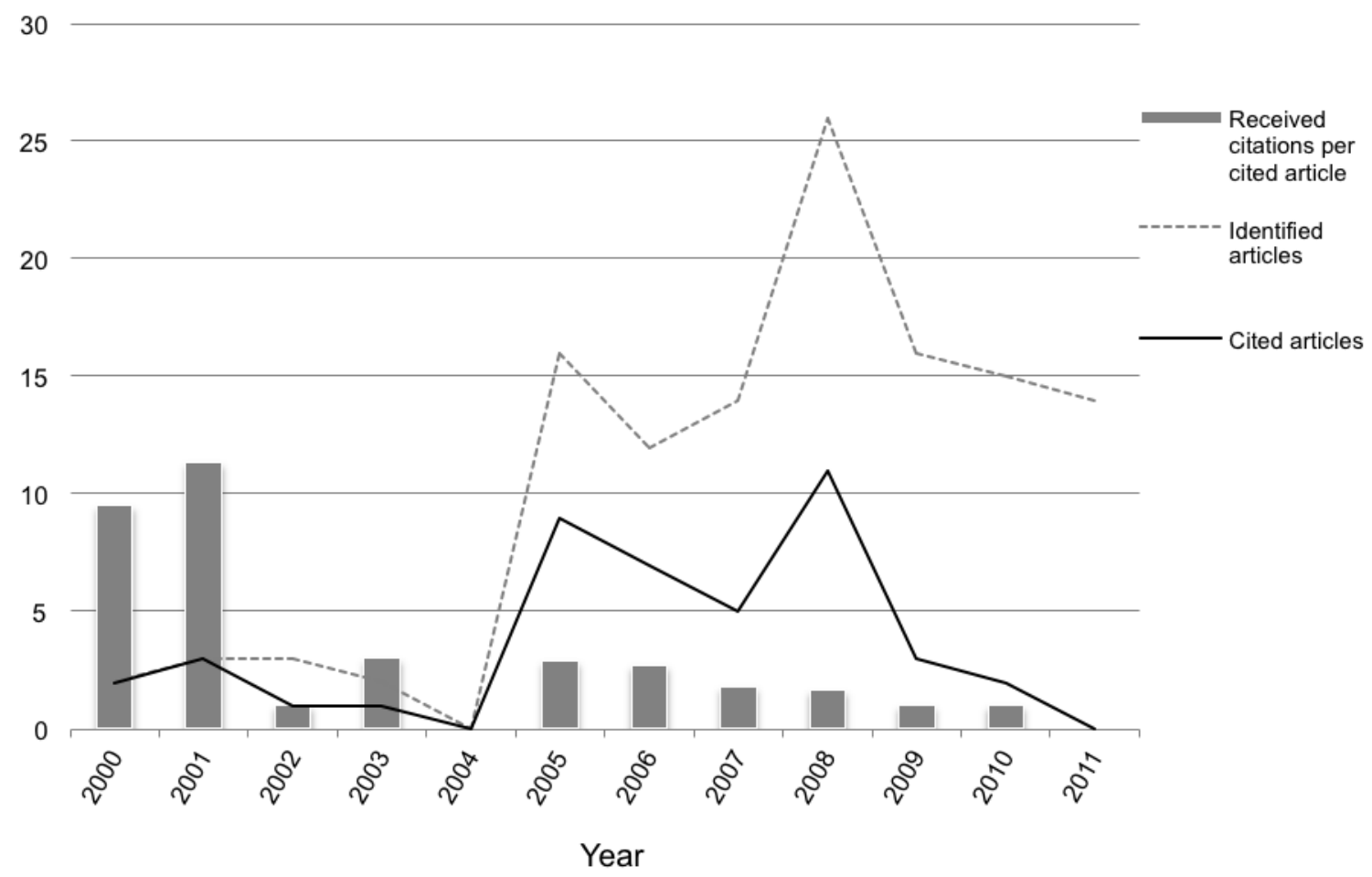

Figure 5: Number of identified and cited articles and received citations per cited article.

During the following period from 2002-2004, the published articles received significantly less acknowledgement: only two articles out of five were cited with a total number of four citations. The period from 2005-2008 experienced an increase in the number of published articles and correspondingly, the number of these articles that received citations also increased (32 out of 68). This resulted in an increase of the number of citations (72) with an average of 2.2 per cited article. Finally, the most recent period (2009-2011) presents five articles that have each been cited once. Essentially, it can be said that the trend of acknowledgement measured as the number of citations per article referenced in journals has been declining steadily over time. However, this trend could be explained by the lag between the moment a paper is published and when citations, especially citations referenced in journals, start to appear. A similar behavior can be observed when analyzing the last column of Table 5, which shows the received citations of the 44 cited articles collected from Google Scholar. The cited articles have received an average of 30.4 citations in Google Scholar with a standard deviation of 30.9.

\subsection{RQ3}

What are the most investigated research topics and their trends over time?

Before answering this question, an analysis concerning the target variable of the research has been performed previously. As many as 91 papers (70.5\%) focus on the effort variable, which usually becomes the dependent variable of the analysis. From them, only seven 
papers $(5.4 \%)$ share this focus with another target variable (three papers share with duration and the other four papers share with size, productivity, defects, and team size). Fig. 6 presents the distribution of papers (29 papers, 22.5\%) that do not focus on effort and instead center their research in a construct or variable different from effort, such as productivity, defects, duration, etc. Conversely, nine papers (7\%) do not focus on any target variable.

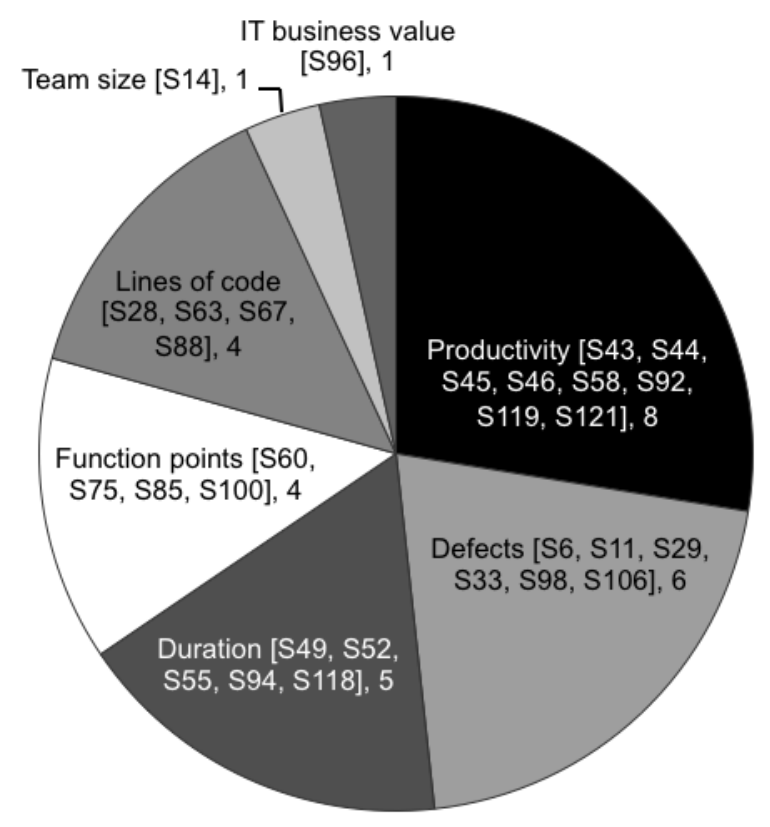

Figure 6: Further considered variables apart from effort.

ISBSG is more suitable for research related to effort and productivity rather than defects for several reasons. The ISBSG dataset describes software quality in terms of the number of defects delivered and reported in the first 30 days of operation of the software. This total number is broken down into three columns corresponding to minor, major, and extreme defects [S6]. Since other defects might have been reported after the first month, the use of only a 30 day interval after the release may constitute the first limitation. Finally, the variable defect density is defined as the defects delivered in a software unit. These variables, however, contain a significant number of missing values, substantially more than for effort-related variables. To overcome this, some authors have worked with the sum of minor, major, and extreme defects recorded [S98] and a successful improved methodology based on the k-NN multiple imputation, Monte Carlo simulation, and stepwise regression has been reported [S29, S33, S106]. Another limitation is the lack of normality of these variables, even after log-transformation, which requires the use of non-parametric methods. In any case, some learning algorithms (i.e. Regression via Classification) have been employed to generate comprehensible models of software defects [S98]. This way, the nature of the data about defects in the ISBSG dataset may explain, to some extent, the reduced number of defect-related papers in the set of analyzed papers compared to those dealing with variables effort or productivity. 
Taking into account that a paper can be classified in more than one research topic, Table 6 shows the distribution of topics following the classification of Jorgensen and Shepperd in [15]. With respect to this classification, the category Phase effort estimation has been included (originally in Other topics) due to its relevance in the set of papers analyzed. Effort distribution by phase is an important aspect in the cost estimation process [16].

Table 6 suggests that the most common research topic is Estimation methods (70.5\%). A paper that shows effort estimations has been classified in this category. Considering that 29 out of 129 papers focus their research on a variable different from effort, this percentage could be even higher if only the papers that focus on effort were taken into account (100\%).

Table 6: Number and proportion of papers for each research topic.

\begin{tabular}{|c|c|c|}
\hline Research topic & $\begin{array}{l}\text { Number } \\
\text { of papers }\end{array}$ & $\begin{array}{c}\text { Proportion } \\
\%\end{array}$ \\
\hline Estimation methods & 91 & 70.5 \\
\hline Dataset properties & 47 & 36.4 \\
\hline Calibration of models & 32 & 24.8 \\
\hline Production functions & 18 & 13.9 \\
\hline Size measures & 15 & 11.6 \\
\hline Organizational issues & 11 & 8.5 \\
\hline Measures of estimation performance & 6 & 4.6 \\
\hline Phase effort estimation & 4 & 3.1 \\
\hline Effort uncertainty assessments & 2 & 1.5 \\
\hline Other topics & 21 & 16.3 \\
\hline
\end{tabular}

The second largest category is Dataset properties with 47 papers (36.4\%). It has been generally recognized that preliminary data analysis is necessary for software cost estimation [17] because the effectiveness of historical datasets in the generation of estimation models depends critically on the nature of the data within the dataset. For this reason, how to analyze datasets for the purpose of estimation methods when based on a historical dataset is an important issue. In this regard, most of the identified references try to somehow tackle this problem before working with an estimation method. Nevertheless, the category Dataset properties includes articles that are specially devoted to data preprocessing in order to minimize the impact of ambiguities, wrongly recorded values, 
missing values, unbalanced datasets, etc., on the quality of any statistical or data mining technique applied to these type of repositories [S58].

In more detail, clustered data and missing data are the most investigated issues in this category as can be seen in Fig. 7. The category Clustered data includes papers that generally try to reduce the heterogeneity of the datasets identifying subsets of project data with a clustering approach in order to form consistent project groups defined by several project properties (even chronologically) to predict the effort. By using this approach, a set of segmented models could be specifically tailored for each of these project clusters. The group Missing data includes papers (14 papers, 29.8\%) that deal with imputation methods (k-NN -Nearest Neighbour Model, modified k-NN, Monte-Carlo simulation, ensemble imputation methods, etc.) to solve this quality data problem. Another subgroup is formed by papers ( 6 papers, $12.8 \%$ ) focused on the outliers treatment, such as the procedures used to eliminate these outliers and in general identifying the impact of this type of dataset noise on the performance and robustness of the estimation methods. Finally the Others category (10 papers, $21.3 \%$ ) collects topics that include the identification and selection of dominant factors and more specifically those papers that present several treatments in dataset preprocessing making it difficult to consider a predominant one.

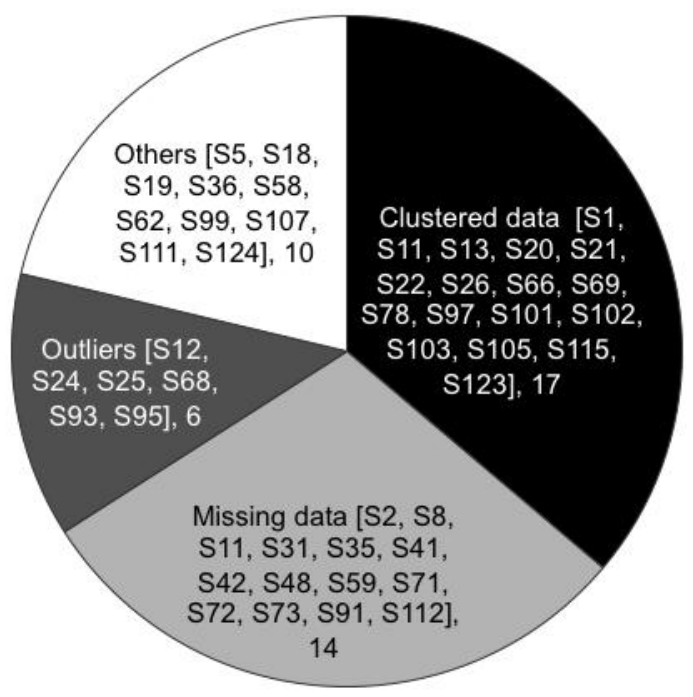

Figure 7: Dataset properties.

Finally, Fig. 8 shows the evolution over time of the three predominant topics, Estimations methods, Dataset properties, and Calibration of models, listed accordingly in Table 6. 


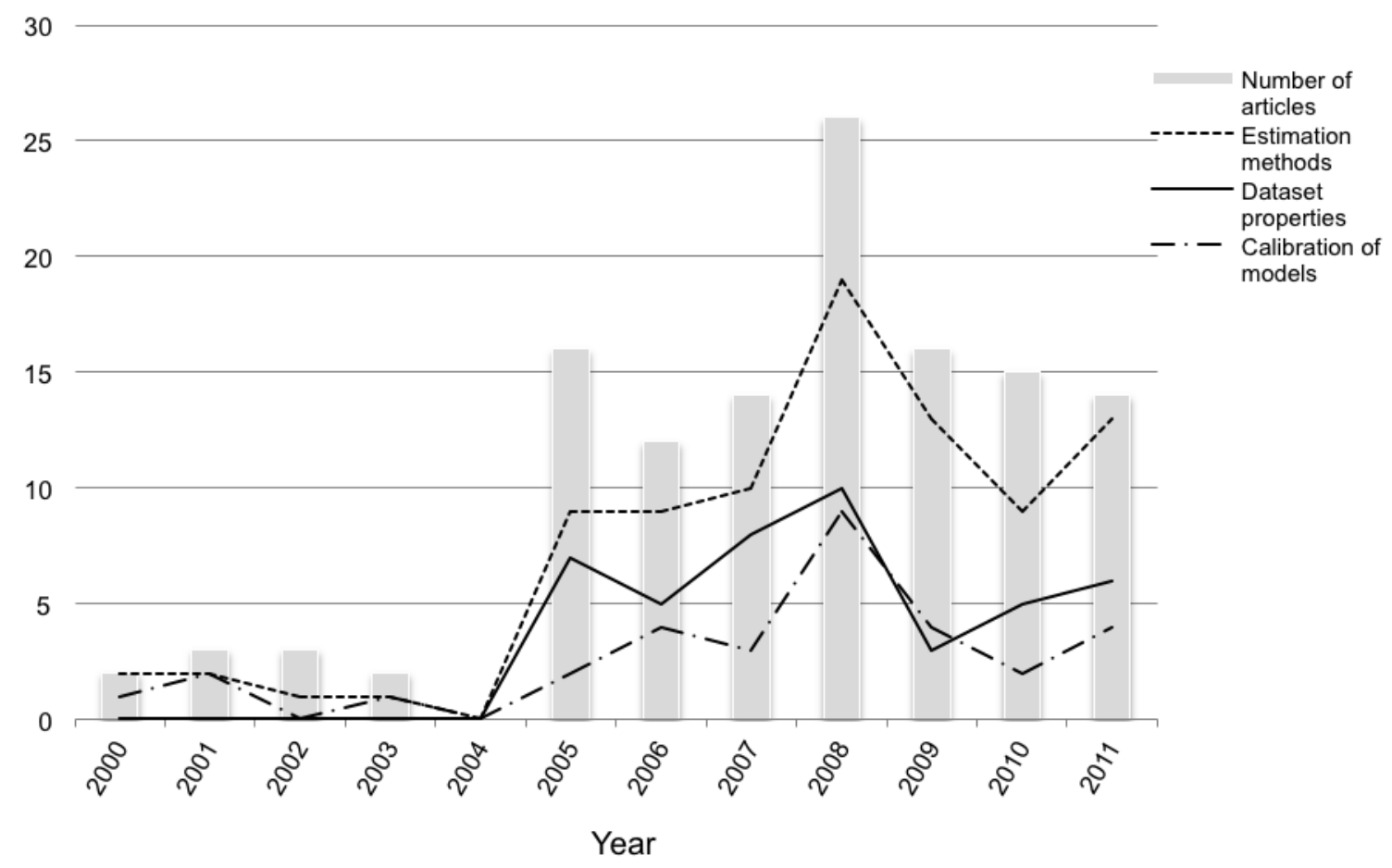

Figure 8: Evolution over time of the three most frequent topics.

In the first period (2000-2004) with a very limited number of articles (10), the two predominant topics Estimation of methods (6 papers throughout the period) and Calibration of models (4 papers) encompass all of the references with no papers classified in the group Dataset properties. The category Dataset properties begins to noticeably appear starting in the year 2005 and appears to have even more relevance than the topic Calibration of models. During the period from 2005 to 2011, the most common research topic is by far Estimation methods, followed by Dataset properties, and then Calibration of models. During 2010, there is a renewed interest by researchers in estimation methods and dataset properties.

\subsection{RQ4}

What are the most investigated estimations methods and trends over time?

Table 7 presents the methods that have been used in ISBSG data analyses and the number of references in which such methods appear, considering that a single paper may have used several methods. Seventy-nine articles of the set have used regression making it the most frequently used method. This result is consistent with other authors and this method is considered to provide good accuracy ([S15], [15]). The Regression group includes a wide range of regression-based estimation models including linear regression, multiple linear regression, ordinary least-squares (OLS) regression, stepwise regression, robust regression, ordinal, categorical and logistic regression, multivariate adaptive regression, ANOVA, and ANCOVA to name a few. Occasionally, this method is used to 
contrast the results that have been obtained by other methods. Another group that has been identified as predominant is made up of the machine learning oriented methods, which have been used with at least one of these techniques by 46 references $(35.7 \%)$.

The third method is Estimation by Analogy (EbA). Together with case-based reasoning estimation methods, both may be viewed as a systematic development of the expert opinion through experience learning and exposure to analogue case studies. The aim is to compare the software project being developed with similar historical projects for deriving software effort estimates [18,19, S63]. This group includes 29 references representing $22.5 \%$ of total.

The category Combination of Estimates includes studies on the combination of estimates from different sources (13.2\%). Additionally, there is a group where the estimation method used has not been relevant ( 7 references) due to the goals and procedures of these papers. A new group defined as Fuzzy has been included. This group was included in the Others category for the Jorgensen and Shepperd classification [15]. Papers belonging to that group have used estimation methods based on fuzzy systems. A fuzzy system usually consists of a combination of a set of logical expressions with fuzzy sets used to deal with linguistic terms that derived after partitioning a dataset to a number of intervals and provide a representation scheme for dealing with uncertain, imprecise, and vague concepts [20,21]. For example in [S103], fuzzy clustering is used for segmented models. Fuzzy methods are usually used in combination with other methods.

Finally, there is also a group that includes an extensive variety of methods with 18 papers (13.9\%) called Others that conveys the high diversity of estimation approaches [15]. This category includes survival analysis, multiple criteria linear programming, production function analysis, sequential quadratic programming, case studies, etc.

In contrast to Jorgensen and Shepperd results [15], no papers have been found dealing with the following methods: Expert Judgment, Work Breakdown, and Theory basedestimation approaches. This is because ISBSG does not include attributes that would support such types of research. It should be mentioned that it is valuable to complement the information obtained from data driven techniques with expert judgment in order to develop estimation methods that take into consideration the expertise component. Usually, methods that make use of all information available in organizations make practical and economic sense [S124]. For example, Dalmazo et al. [S82] merge data from ISBSG with information provided by a group of experienced IT project managers to overcome the missing data problem when building a Bayesian model.

Table 7: Number and proportion of papers for each estimation method.

\begin{tabular}{lll}
\hline Method & $\begin{array}{c}\text { Number of } \\
\text { papers }\end{array}$ & Proportion \% \\
\hline
\end{tabular}




\begin{tabular}{lcr} 
Machine Learning & 46 & 35.7 \\
Estimation by Analogy & 29 & 22.5 \\
Combination of Estimates & 17 & 13.2 \\
Function Point & 8 & 6.2 \\
Fuzzy & 8 & 6.2 \\
Simulation & 3 & 2.3 \\
Not relevant & 7 & 5.4 \\
Others & 18 & 13.9 \\
\hline
\end{tabular}

If the machine learning methods are analyzed in detail, this family of methods includes the approaches described in Table 8.

Table 8: Machine Learning estimation methods.

\begin{tabular}{lcc}
\hline \multicolumn{1}{c}{ Machine Learning method } & $\begin{array}{c}\text { Number } \\
\text { of papers }\end{array}$ & $\begin{array}{c}\text { Proportion } \\
\%\end{array}$ \\
\hline Neural Networks (NN) & 18 & 13.9 \\
Model tree & 10 & 7.7 \\
Classification and & 9 & 7.0 \\
Regression Trees (CART) & & \\
Genetic Algorithms (GA) & 7 & 5.4 \\
Support Vector Machines (SVM) & 6 & 4.6 \\
Bayesian Networks (BN) & 5 & 3.9 \\
Association Rules (AR) & 3 & 2.3 \\
Others & 16 & 12.4 \\
\hline
\end{tabular}

Many references have used more than one of these methods and therefore, the total sum exceeds the 46 references mentioned above in Table 6 . Within the Machine Learning category, the most frequently used method is Neural Networks, followed by Model Tree, Classification and Regression Trees, and Genetic Algorithms. A significant number of methods related to this category could not be classified conveniently so they were placed in the group Others. It should be mentioned that two papers within this group have used ensembles of Learning Machines. 


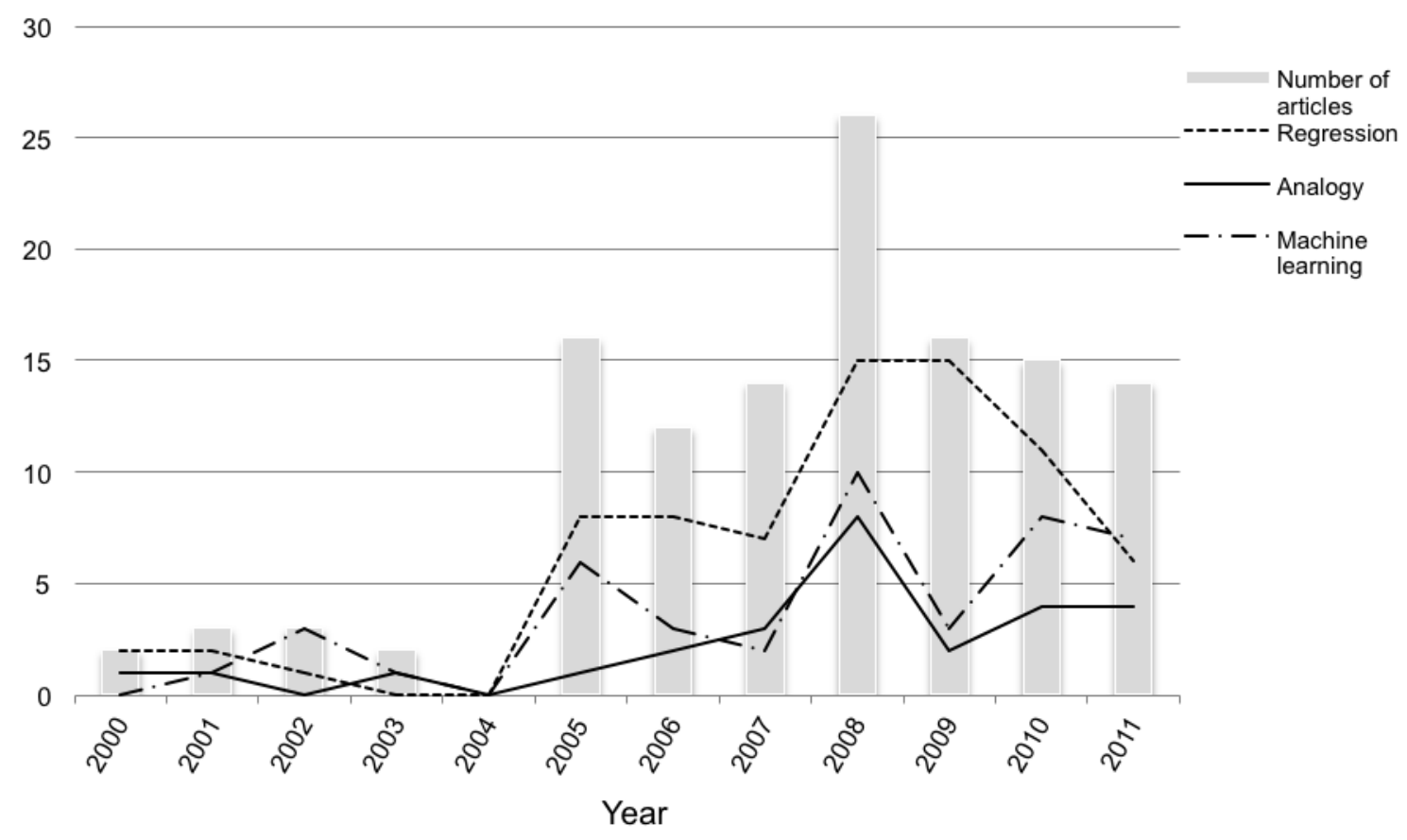

Figure 9: Evolution over time of the three most frequent estimation methods used.

Fig. 9 shows the trend over time of the three main groups of estimation methods:

Regression, Machine Learning, and Estimation by Analogy methods (EbA). The estimation methods are superimposed over the number of articles published each year. It is important to note the predominance of regression methods over time, although a slight decline can be observed since 2009. Also, a boom of EbA methods during the 2007-2008 period (30.8\% of the published papers) with a subsequent decline can be observed [15]. Lastly, the increasing presence of Machine Learning-based methods that also have a peak within the $2007-2008$ time period (38.5\%) and consolidate their presence in the identified references can be seen.

Further analysis has been performed trying to relate trends in the usage of the more relevant estimation methods with ISBSG data to trends of these methods in the broader field of empirical software engineering. Hence, the abstract and citation database, Scopus, has been used to track the number of papers that have dealt with these estimation methods in all 19 journals included in Table 2. The analysis of these journals enables us to get a valid perspective on the field of empirical software engineering. The terms searched in the article title, abstract, or keywords were: "regression," "analogy," and "machine learning." The results obtained are shown in Fig. 10. 


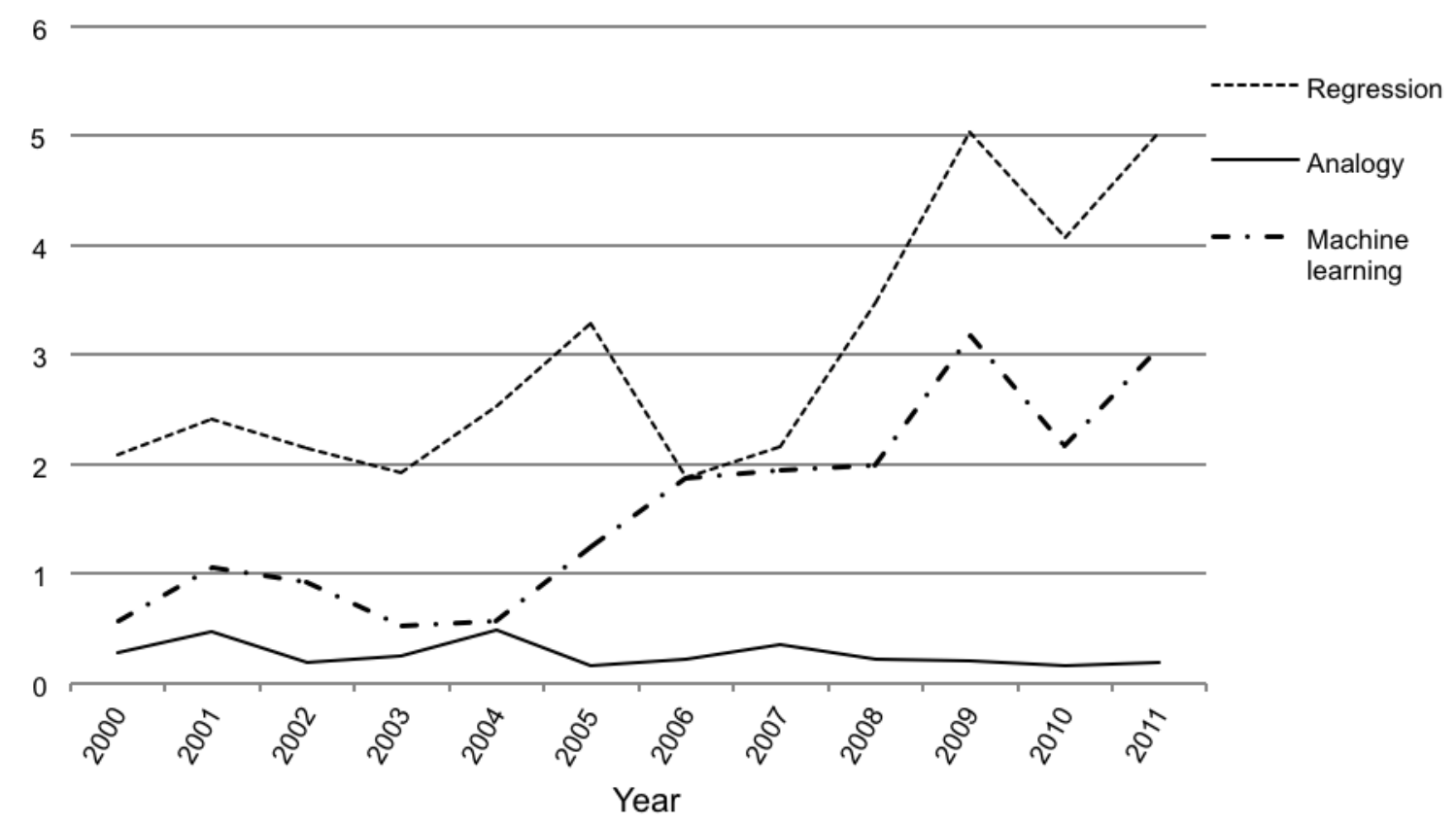

Figure 10: Evolution over time of the three most frequently used estimation methods in journals with ISBSG-based research papers.

Fig. 10 depicts the percentage of papers that have worked with these methods over the total number of papers published in the mentioned journals. The trends of the more relevant estimation methods with ISBSG data somehow follow the trends obtained in the broad research field. Regression is the predominant method followed by machine learning and analogy-based estimation. There is a rising trend in regression and machine learning methods with a maximum value occurring around 2009 while analogy-based estimation methods show a more constant behavior. As an aside, the relative presence of analogybased methods is less relevant in the realm of empirical software engineering than in ISBSG-based research. Moreover, Fig.9 shows a decrease in the use of Regression and Machine Learning methods, which is not observed in the field of empirical software engineering.

\subsection{RQ5}

Is ISBSG data a valuable source of data for the research undertaken in the paper?

Seventy-one identified references (55\%) support their research exclusively with ISBSG data while 58 papers use additional data. Fig. 11.a shows the development of the ISBSG dataset from release 4 (1997) to release 12 (2013) as well as the date each release was delivered. It is apparent that the usual interval of appearance for each release is fewer than three years except for R9 and R11, which were in place for three and four years respectively. The repository has grown from 2,000 projects out of 16 countries in 2003 (R8) to 6,006 projects included in release $12[1,22]$. The number of references that have used each ISBSG release is presented in Fig. 11.b. The most frequently used ISBSG 
release is release 10 with 32 references, but release 7 (25), release 8 (22), and release 9 (23) are also oftenly used. To interpret the results of release 11 (5), it is important to keep in mind that the papers were collected until the end of June 2012.
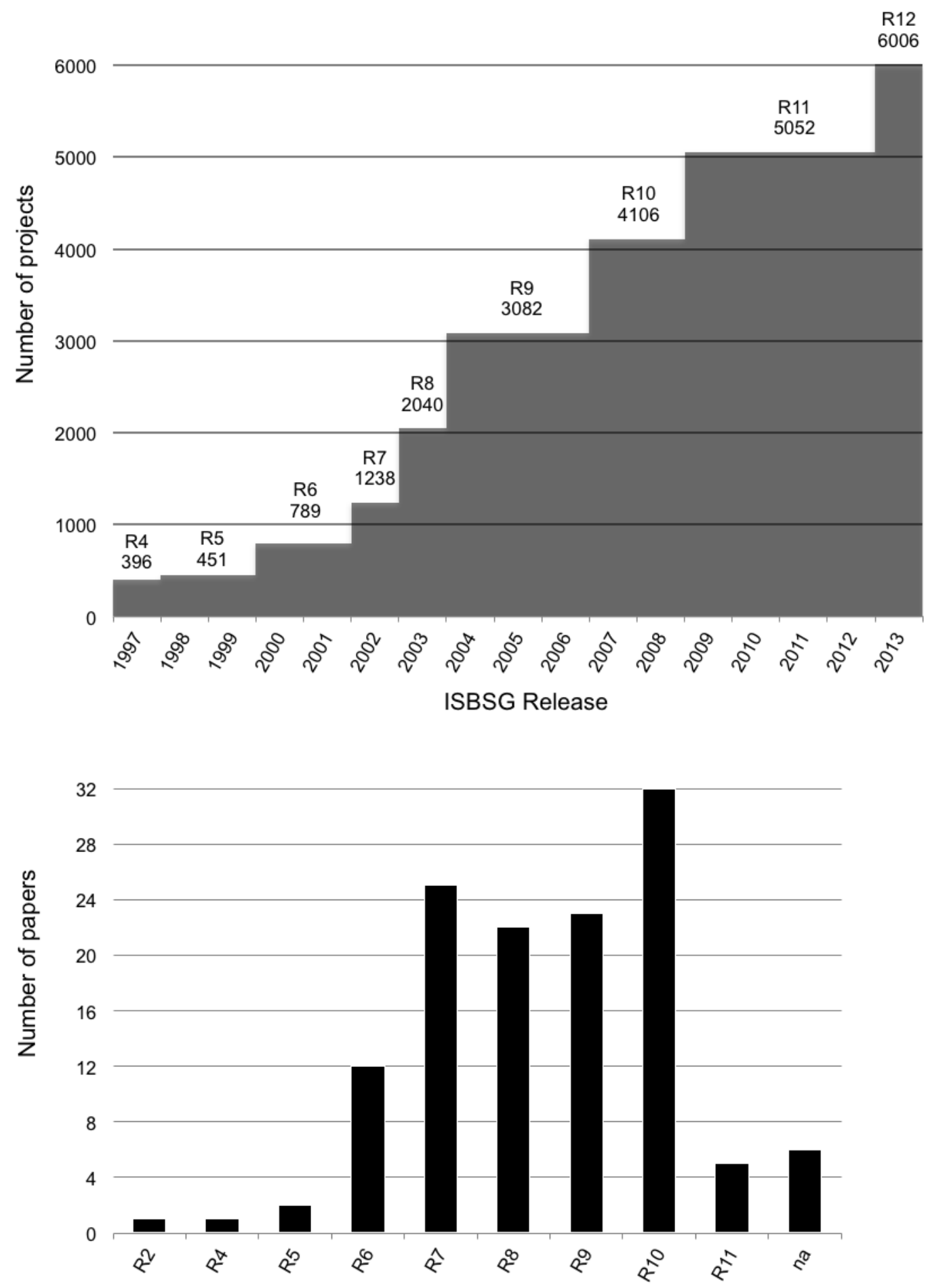

ISBSG Release 
Figure 11: ISBSG releases and the evolution of the number of papers using a specific release of ISBSG. (a): ISBSG releases over time. (b): Evolution of the number of papers using a specific release of ISBSG.

Most of the papers (43) that use additional data only employ other datasets that are in the public domain or have a limited usage [23]. Datasets with a limited usage do not allow researchers to publish the dataset in its entirety or give the dataset to other parties without the owner's consent because organizations pay an annual fee to gain access to the data. This is the case for the Finnish (also called the Experience dataset) or CSBSG datasets.

To summarize, as many as 30 datasets are used, but only 13 are used more than once. Table 9 shows information about the most referenced datasets including the number of papers that use them, their source, whether they are public or of limited usage, and whether or not they are included in the PROMISE repository. It is worth noting that ISBSG has agreed to include some of its data in the PROMISE repository with one small subset appearing in late 2012.

Table 9: Other datasets used apart from ISBSG.

\begin{tabular}{lcccc}
\hline \multicolumn{1}{c}{ Dataset acronym } & Source & $\begin{array}{r}\text { Number of } \\
\text { papers }\end{array}$ & $\begin{array}{c}\text { Public } / \\
\text { Limited }\end{array}$ & PROMISE \\
\hline Desharnais & {$[24]$} & 20 & $\mathrm{P}$ & $\mathrm{Y}$ \\
Cocom081 & {$[25]$} & 14 & $\mathrm{P}$ & $\mathrm{Y}$ \\
STTF = Bank63 & {$[17]$} & 14 & $\mathrm{P}$ & $\mathrm{Y}$ \\
Kem87 & {$[26]$} & 13 & $\mathrm{P}$ & $\mathrm{Y}$ \\
Albrecht (\& Gaffney) & {$[27]$} & 12 & $\mathrm{P}$ & $\mathrm{N}$ \\
Nasa93 = Cocnasa & {$[28]$} & 8 & $\mathrm{P}$ & $\mathrm{Y}$ \\
USP05 & {$[55]$} & 7 & $\mathrm{P}$ & $\mathrm{N}$ \\
Mendes03 & {$[29]$} & 5 & $\mathrm{P}$ & $\mathrm{N}$ \\
Finnish = Experience & {$[30]$} & 4 & $\mathrm{~L}$ & $\mathrm{~N}$ \\
Sdr & {$[28,31]$} & 4 & $\mathrm{P}$ & $\mathrm{Y}$ \\
Abran \& Robilard & {$[32]$} & 3 & $\mathrm{P}$ & $\mathrm{N}$ \\
CSBSG & {$[33]$} & 2 & $\mathrm{~L}$ & $\mathrm{~N}$ \\
China & {$[28]$} & 2 & $\mathrm{P}$ & $\mathrm{Y}$ \\
\hline
\end{tabular}


On the other hand, only 15 of 58 papers make use of private data. Some use a few projects such as case studies [S70, S88, S14] while [S37] and [S126] use data from leading information technology companies and organizations, Commercial off-the-shelf (COTS) package builders, academic and professional books, and technical reports.

Other authors use private datasets such as the Israeli dataset, which consists of projects from several high-tech (mostly software) companies [S47]; the Teletel dataset from a Greek telecom software company [S39], the Megatec dataset from an Australian software developer [S4], the Bank dataset from the Turkish subsidiary of a multinational bank [S20] and the Bank dataset from a financial company located in Korea [S24, S78]. In [S82] a synthetical project dataset, known real data is merged with information provided by a group of experienced IT project managers.

Only two papers combine private data with other datasets apart from ISBSG. Therefore, 45 papers use ISBSG with other non-private datasets. Within this set, $46.7 \%$ of the papers use two datasets including ISBSG, $51.1 \%$ use between three and nine datasets, and one paper uses twelve datasets.

\subsubsection{Reasons for the usage of complementary datasets}

The motivation behind the usage of these complementary datasets is summarized in the following:

- To evaluate the performance of an estimation method [S2, S70, S114] and identifying the datasets that favor such method. In software effort estimation models, the characteristics of datasets affect the results and the overall procedure. These characteristics are considered major factors in assessing the performance of the proposed techniques [S43, S45, S76, S83]. Consequently, the structure of the dataset itself should be taken into account before judging which dataset is suitable for a particular method [S71]. For example, the predictive accuracy and the model fitting depend on the type (quantitative or non-quantitative) and number of project attributes [S3, S86], the sample size [S30, S66], or the missing data [S3, S48, S59, S73]. Consistently, 32.8\% (19 out of 58) of the papers that have used complementary datasets were previously classified in the category Dataset Properties when the research topic was considered (RQ3) and $40.4 \%$ of the papers in this category have used other datasets to assess the influence of the nature of their data in the performance of software estimation models.

- To confirm or disprove the hypothesis that there is a difference in accuracy between estimates derived from multi-company data such as the ISBSG dataset and estimates derived from company-specific data [S4, S24, S99]. Furthermore, it is necessary to gain more insight into heteroscedasticity (non-uniform variance); a problem that affects datasets that combine data from heterogeneous sources [S21, S96].

- To confirm the reliability of the proposed method [S9, S12, S68, S76, S86]. A wider application to more datasets could generalize the results and reveal more insight 
into the methods. Indeed, papers that make use of fewer datasets consider this as a limitation and propose to prevent this weakness by applying the method to other datasets and comparing their effects [S19, S24, S25, S30, S72, S78, S107].

Furthermore, there are some obvious reasons to use complementary datasets that are not usually explicit in the papers, but should also be considered. For example, complementary datasets should be used when they have been used by previous studies with a similar goal [S46, S77] or simply because complementary datasets are available to the authors.

\subsubsection{Advantages and limitations related to the usage of ISBSG}

The ISBSG data can be used for different purposes, such as evaluating the benefits of changing a software or hardware development environment, improving practices and performance, or estimation [S12, S51]. The ISBSG dataset has been used by many studies focused on the issue of software estimation in spite of the diversity of its data elements [S24, S65]. The main advantage obtained from the use of the ISBSG dataset is that it offers a wealth of information regarding practices, tools, and methodologies accompanied by process and product data to be used in benchmarking, monitoring, control quality, and performance management purposes during the software development process [S80]. Besides, the dataset holds data for projects that address feasibility, planning, and requirements analysis and for projects that do not address the complete lifecycle [S14].

External validity is the ability to generalize obtained findings outside the specifications of the study ([S66], [34]). External validity suffers from some restrictions that include the degree to which the selected attributes describe the projects and the representative capacity of datasets [S72]. ISBSG contains an analysis of software costs for a group of completed projects that come from a broad cross section of industry which differ in size, effort, platform, language, and development techniques data [S12, S41, S90, S107]. As a consequence, the ISBSG dataset covers a wide range of possible project attributes [S3] and application environments. Some public datasets, still widely used for comparison purposes, are too old to be used in software cost estimation because they represent nonrelevant software development approaches and technologies [S111]. This is not the case with the ISBSG dataset, which continues to grow over time to provide valuable information. This dataset, however, represents the more productive projects in the industry, rather than industry standards, because participant organizations are considered to be among the best software development houses [S41] and also, they may have chosen to only submit their best projects rather than typical ones [S90]. Therefore, the projects have not been selected randomly and the dataset is more than likely subject to biases [S107].

Public dataset repositories provide an opportunity for diminishing data collection costs that are an expensive and time-consuming process for individual organizations [S4]. Besides, cross-company datasets are beneficial for the small-sized companies that are not experienced in data measurement [S99]. This way, a company can select, isolate, and analyze suitable data and projects, and estimate its own new projects based on its needs 
[S39]. Furthermore, datasets that are not publicly available make studies unreproducible ([S51], [35]).

There is a debate over whether multi-organizational data is valuable for estimation [S4, S24, S99]. The ISBSG data is collected from various worldwide organizations with dissimilar backgrounds, business cultures, levels of personnel experience, and development maturity [S5, S21, S67, S107]. As a result, the dataset is widely scattered and inconsistent, which effectively presents the problem of divergences of variances [S21, S71]. This portrays a clear limitation of data repositories such as the ISBSG dataset. This large variation of data distribution, however, is also one of the characteristics of the data collected from single-company with an unstable software process [S24]. In any case, heterogeneity makes it difficult to obtain accurate predictions [S4, S24, S77, S78]. On the other hand, cost estimation includes many statistical methods (regression, ANOVA, etc.) whose basic assumption is the independence of projects. This premise is satisfied in large cross-organizational datasets since projects come from different companies around the world [S17].

Experiments with ISBSG enable the comparison between estimation method performances [S2]. Since ISBSG projects used for calibrating estimation models may be different than the types of projects under consideration, this may be a potential source of error [S87]. Furthermore, multi-organizational datasets are not easily usable for noncontributing companies to establish their effort estimation models [S80]. In case it is necessary to predict effort by using the ISBSG repository for a non-contributing company, OLS regression should be considered rather than analogy [S4]. Besides, models occasionally would need to be recalibrated on newly collected data, as relationships between attributes can change over time [S51].

The effectiveness of historical datasets in the generation of prediction models is critically dependent on the nature of the data within the dataset [S84, S124]. Therefore, the dataset quality and variance are influential factors. Since the ISBSG repository is a large heterogeneous dataset, a data preparation process is required to obtain the appropriate subset for analysis [S24, S49, S98, S114]. This is consistent with the ISBSG guidelines that suggest basing the analysis on a suitable subset of projects [S11, S19, S83].

First of all, the dataset should undergo a data cleaning process based on quality criteria. Fortunately, project entries in the dataset are validated by the ISBSG organization against specific quality criteria [S4] for completeness and integrity. A quality rating for the credibility of each project is given in order to achieve the grade of usefulness of the data for various analyses [S30, S107]. Usually the projects with low data quality ratings, i.e. C or D, are excluded from analysis [S87, S88].

Next, the most representative data should be identified and selected while attributes and projects with no direct or apparent effect on software estimation are ignored [S45, S107]. For example, in [S21], the size measurements are considered the main driver of project effort, so the dataset is cleaned for homogeneity for this aspect. This selection process 
can be supported by the normality and correlation test and data normalization [S24]. In most cases, features are selected using brute-force and other forms of search heuristics. Since this requires a large amount of computing power and time, it is especially problematic when dealing with large datasets [S65]. Moreover, generally, learning from a smaller dataset, containing a limited set of highly predictive attributes, is easier than learning from a bigger, noisier dataset containing many redundant or irrelevant attributes. This way, the resulting models will contain less attributes and will be also more stable since potential collinearity between attributes will be reduced. Thus, a more concise model is easier to interpret and preferred over a more complex model [S51].

ISBSG is a dataset with projects characterized mostly by a mixture of categorical [S87, S114], continuous, and discrete attributes [S41]. The dataset contains many missing and outlier values [S76]. Handling incomplete data is an important issue faced by researchers who use industrial and research datasets. This is the case within ISBSG, which suffers from a large amount of missing data requiring imputation and other missing value techniques [S44, S65, S114]. Listwise deletion, however, has been the most common way of dealing with missing data. Projects that have missing values for any effort driver are usually excluded from the dataset for modeling [S90]. In ISBSG, the large number of missing values in almost all of the important variables can result in a significant reduction of the data used to build the model [S19, S114], leading to a severe loss of statistical power. It is also argued that removing projects with missing values could risk losing valuable information [S76].

On the other hand, a multi-company dataset such as ISBSG also suffers from the presence of more outliers in comparison to a single-company dataset [S24]. In general, the presence of outliers in a dataset tends to increase the value of the standard error of estimation [S47]. Furthermore, there is neither data related to the experience of the software developers, nor any parameter that identifies the company or gives information about the organization type of the company in the repository [S4]. Consequently, it is more difficult to form homogeneous subsets which can result in significantly more accurate estimates.

Ultimately, data partitioning is considered necessary in preprocessing due to data variance [S43]. In this sense, the high number of projects makes it possible to prune the ISBSG dataset into subsets with several requirements [S2, S21, S73, S90]. For example, in [S24], the authors consider it better to use the dataset after categorization according to similar characteristics, such as business domain, in order to obtain a more accurate estimation result. In [S9], a new cross-domain dataset is formed from the ISBSG dataset by selecting the projects whose application types are not embedded. Although homogenous datasets can be formed from the original one, projects in the ISBSG dataset still have greater variation in system size, development effort, and productivity [S70]. Furthermore, the estimation results obtained from the ISBSG model are not so promising when compared to organization's own estimation model results [S80]. To overcome the effect of heterogeneity, some authors have eliminated extreme points [S21]. 


\section{Discussion}

This section summarizes the principal findings of this systematic mapping. It also includes the limitations of the study and discusses the implications for researchers and practitioners.

\subsection{Principal findings}

This study shows the extent to which and how ISBSG has been used by software engineering researchers until June of 2012. Thereby, the papers that have worked with this dataset have been identified and classified by answering a set of research questions. This systematic mapping review conveys a picture of the potential and limitations of ISBSG as a research facility.

The search term "ISBSG" was the input for four bibliographic databases: IEEE Xplore, ACM Digital Library, ScienceDirect, and Web of Knowledge. This resulted in 134, 63, 57, and 52 results respectively. A complementary search was performed within the journals only indexed in the Web of Knowledge, and as a result, 21 additional papers were included. The search was completed in June 2012. After the elimination of duplicates and the filtering process, the most relevant information was obtained from each of the 129 remaining studies.

Sixty-two of the remaining 129 papers are articles published in 19 journals. Forty-eight percent $(48.4 \%)$ of papers are included in the Journal of Systems and Information (11 papers), Information and Software Technology (10 papers), and Empirical Software Engineering (9 papers) journals. The conference papers (67) were presented in 40 conferences. PROMISE, METRICS, and ESEM encompass $26.9 \%$ of the identified conference papers. The first papers were published in the year 2000 and following an introductory period, there was a period from 2005 to 2011 with a nearly constant total presence of this type of research either in conferences or journals, with an average of 16 publications, reaching a maximum of 26 references in 2008.

There are 44 identified papers (35.8\%) in the period 2000-2011 that have received at least one citation in journals. Only five papers have received six or more citations. All in all, they have received 134 citations. ISBSG holds a list with the research papers that have used, or refer to, the ISBSG repositories. This list contains as many as 45 references. Only 12 papers of the total 129, however, have been submitted to the ISBSG list. Besides, it can be noted that some references listed have not been retrieved in the collecting process explained in this paper. This fact is justified because the references that have not been retrieved are, in most cases, documents that were not considered here, i.e., PhD thesis. Greater efforts should be completed to promote and update the ISBSG list. Also, researchers should always contact ISBSG after publishing any relevant work.

As many 91 papers (70.5\%) focus on effort variable and in a few cases (5.4\%) they share the focus with another target variable. Twenty-two percent $(22.5 \%)$ of the papers center their research in a construct or variable different from effort and $7 \%$ do not focus on any 
target variable. The most common research topic is Estimation methods (70.5\%). The second largest category is Dataset properties (36.4\%), which begins to appear noticeably from the year 2005. In more detail, clustered data and missing data are the most investigated issues in this category. From 2010, there is a renewed interest of researchers in estimation methods and dataset properties.

In regards to the estimation methods, it is important to note the predominance of regression methods over time $(61.2 \%)$ followed by machine learning $(35.7 \%)$ and estimation by analogy (22.5\%) estimation methods. These final two methods present a renewed presence since 2008 meanwhile the interest in regression methods seems to have declined slightly. Within the Machine Learning category, the most used methods are Neural Networks, followed by Model Tree, Classification and Regression Trees, and Genetic Algorithms.

Finally, 71 identified references (55\%) support their research exclusively using ISBSG and 58 papers also use additional data. The most used ISBSG release is release 10 with 32 references. Most of the papers (43) that use additional data, employ other datasets that are in the public domain or have a limited usage. All in all, as many as 30 datasets are used, but only 13 are used more than once. On the other hand, only 15 out of 58 papers make use of private data. The principal reasons, derived from the analysis of these 58 papers, for the motivation behind the usage of complementary datasets are: to compare the performance of an estimation method, to confirm or disprove the hypothesis that there is a difference in accuracy between estimates derived from multi-company data and estimates derived from company-specific data, and to confirm the reliability of the proposed method. Additionally, in many papers (32.8\%), complementary datasets have been used to deal with problems related to the dataset data properties.

Public dataset repositories provide an opportunity to diminish data collection costs that are an expensive process for individual organizations. The main benefit obtained from the use of the ISBSG dataset is that it offers a wealth of information regarding practices, tools, and methodologies accompanied by process and product data to be used in benchmarking, monitoring, control quality, and performance management purposes during the software development process. This body of knowledge enables learning from experience through analysis and research, and disseminating the lessons learned.

Some of the ISBSG drawbacks include that the best projects have been selected and the dataset most probably is subject to biases. Since the ISBSG repository is a large heterogeneous dataset, a data preparation process is required to obtain the appropriate subset for analysis. First, the dataset should undergo a data cleaning process and the most representative data should be selected [36]. ISBSG also suffers from a large number of missing data which requires imputation and other missing value techniques. Ultimately, the partitioning of data is considered necessary in preprocessing due to the variance of data.

\subsection{Study limitations}


It is important to consider that the results obtained from a systematic mapping study could be affected by the researchers conducting the review, by the selected search term, and by the chosen time frame [13]. Some limitations of this systematic mapping have been identified and are discussed below.

The first limitation concerns the search strategy employed. In an attempt to address this limitation, detailed information regarding the specific search term and databases used was reported previously in Section 2.2. On one hand, this work is based on automatic searches performed using the native database search engines, which means some papers might have been lost. This threat, however, was mitigated by selecting search engines which have been considered as the most important scientific sources and therefore are supposed to contain a majority of the relevant studies [37]. The searches were also crosschecked by the authors of this study. To further mitigate this threat, references of each document in the set of selected papers should have been considered using the ISBSG dataset.

On the other hand, a broad overview of the research area was desired, thus no limits were placed on date of publication and the number of restrictions was minimized on primary study selection to be as inclusive as possible. No logical operators were used and the unique search term ('ISBSG') was applied not only to the papers title and abstract, but also to the body of the text. By using this method, it is likely that most of the relevant papers have been included. At this point, it is appropriate to clarify that the search within the full document record was not possible in papers indexed in the Web of Knowledge. Consequently, an additional search was performed within the journals only indexed in the Web of Knowledge, using their publisher's search engine. Then, further work was performed to dismiss those articles that only mention ISBSG or use it as an example and in turn, select only the papers that use ISBSG as a primary source of data (i.e., dataset) as was mentioned in the detailed description of inclusion and exclusion criteria.

It is important to mention that some more recent studies may be missing because the search engines may not have indexed them. Furthermore, the articles were only collected until the end of June 2012. Finally, exclusion of papers written in a language other than English may have lead to a bias in the selection process. This could not be avoided due to the impossibility of the revision team to address these languages.

The second limitation concerns bias in the data collection. The first information collected was about the identification and general details of the paper such as the title, its authors, the paper type, the source where it was published, year of publication, abstract, and keywords. Zotero, a bibliographic management system, was used to collect data and later this information was exported to a standard worksheet format using SQL (Structured Query Language).

Difficulties extracting relevant information from the papers were encountered since many did not provide substantial information in the abstract or keywords to facilitate the analysis 
of the mapping process. In those cases, it was necessary to also read the introduction and conclusion sections and sometimes, even other sections of the primary study, to properly fulfill the data collection form. The data was extracted by the two authors and an additional cross-check was performed.

Another limitation is in regards to the classification of the primary studies. In an attempt to limit this constraint, existing classifications were reused as much as possible. The research topic and estimation method classifications were based on Jorgensen and Shepperd work [15], but it was necessary to adapt them especially for the estimation methods to ensure the latest methods would be included.

Despite using this precaution, the problem still remained and in order to reduce the risk of introducing a bias in paper classification, the classification was performed by the two authors to reach an agreement in problematic cases. It should also be mentioned that English is not the native language of the authors involved in this research.

There is also a limitation concerning research question 2 (RQ2), which presents the number of citations that the papers have received in the dataset because only the citations received in the journals in the dataset have been considered. This problem has been mitigated by contrasting this information with the number of citations that appear in Google Scholar. The fact that some studies were classified in more than one category appears as a potential problem for the descriptive statistics in RQ3. In addition, some of the authors of the identified references used diverse terms to identify the estimation methods because there is no standard terminology (RQ4).

\subsection{Implications for research and practice}

The findings of this systematic mapping study have implications for both researchers who are planning new studies using ISBSG and for practitioners working in the software engineering field.

This study can help researchers make informed decisions about the trending topics, most commonly used variables, and promising methods. This information will help them to assess the originality and potential value of a research proposal. Further, the selected set of papers constitutes a useful background to contrast and learn from previous perspectives. Researchers are also provided with the journals and conferences that, by now, can help them to disseminate their results. In this line, the ISBSG dataset remains interesting and offers potentially valuable research possibilities.

Certainly, the most studied ISBSG variable is effort followed by productivity, defects, and duration. With this in mind, the nature of the data in ISBSG may explain the reduced number of defect-related papers. Also, the most common research topic is by far estimation methods, followed by the study of dataset properties and the calibration of models. In fact, there is a need to continue studying the data nature (ambiguities, wrongly recorded values, missing values, unbalanced datasets, etc.) and their preliminary analysis 
because data features critically affect the quality of any estimation technique applied. Regarding the research methods adopted by the selected studies, regression-based estimation models are the most frequently used and are even used to contrast the results that have been obtained by other methods. Furthermore, machine learning oriented methods including neural networks, model tree, classification and regression tree, genetic algorithms, Bayesian networks, etc. are quite promising not only in dealing with ISBSG data, but also in dealing with the field of software engineering.

This work has also synthesized and described the usual data preparation process including the treatment of missing values and outliers, which is required to obtain the appropriate subset for analysis. Further, the usage of complementary datasets enables the assessment of the validity of estimates derived from multi-company data such as the ISBSG dataset against estimates derived from company-specific data. In general, the application of the proposed method to more datasets could be useful to generalize results, confirm reliability, and to reveal more insight into the method.

This systematic mapping can serve as a starting point in the development of more comprehensive systematic literature reviews and can contribute to a better understanding of the use of the ISBSG dataset by the research community. All of this may foster further research in this area that will provide additional insights to understand and improve the software engineering process.

From a practitioner's point of view, this study presents a selected collection of studies with a wide focus regarding practices, tools, and methodologies to be used in benchmarking, monitoring, control quality, and performance management purposes during the software development process.

This collection of experiences provides practitioners with a real opportunity for diminishing data collection costs through the use of the ISBSG dataset or any other public repository. These costs are always expensive and time-consuming for individual organizations. In this way, software engineering practitioners working for any type of organization can get an overview of existing approaches and possibilities that the ISBSG dataset offers to help them improve their IT resource management by performing their own analysis, estimations (project size, effort, duration, and cost), or benchmarking.

The limitations in the usage of ISBSG are illustrated, in particular, its heterogeneity and the problem with using a multi-organizational dataset for a non-contributing company. Furthermore, learning from a bigger, noisier dataset containing many redundant or irrelevant attributes is more difficult than from a more homogeneous collection of data and a limited set of highly predictive attributes. Additionally, a more concise model is easier to interpret and preferred by practitioners over a complex model with more attributes.

Nonetheless, ISBSG-derived software estimation models provide practitioners with the opportunity to compare their organizations estimation results.

\section{Conclusion and future work}


This work presents the results of a systematic review about the usage of ISBSG until June of 2012. After the searching and filtering process, 129 papers were analyzed coming from as many as 19 journals and 40 conferences. The most relevant journals in terms of number of papers published related to the ISBSG dataset are Journal of Systems and Software, Information and Software Technology, and Empirical Software Engineering. The most relevant conferences include PROMISE and METRICS, followed by ESEM. These papers started to appear in 2000, but have mostly consolidated their presence since 2005. Only one-third (35.8\%) have received at least one citation from journal papers within the set and among the papers that have received more citations, two were presented in METRICS and three were published in the Information and Software Technology journal.

ISBSG is more suitable for research related to effort and productivity rather than defects. The topic studied most predominantly is estimation methods followed by dataset properties, such as clustered data and missing data; both for the purpose of estimation methods and calibration of models. The estimation methods based on regression are predominant, but Machine Learning and Estimation by Analogy methods present a renewed presence since 2008 meanwhile the interest in regression methods seems to have declined slightly. Within the Machine Learning category, the most used methods are Neural Networks, Model Tree, and Classification and Regression Trees. The trends of these estimation methods with ISBSG data follow the trends obtained in the broader field of empirical software engineering.

A total of 45 papers from the set use ISBSG with as many as 30 non-private datasets. The reasons that motivate the usage of complementary datasets include: to compare the performance of an estimation method, to confirm or disprove the hypothesis of a difference in accuracy between estimates from multi-company data and company-specific data, and to validate the reliability of the proposed method. The main benefit obtained from the use of the ISBSG dataset is that it offers a wealth of information regarding practices, tools, and methodologies accompanied by process and product data providing an opportunity for individual organizations to diminish their data collection costs. Nevertheless, in ISBSG the best projects have been selected and the dataset is likely subject to biases. Since the ISBSG repository is a large heterogeneous dataset and also suffers from a large amount of missing data, a data preparation process is required to obtain the appropriate subset for analysis.

In summary, this work presents a comprehensive picture of the existing usage of ISBSG in software development research, which in most cases is related to effort estimation methods. The advantages and limitations related to the usage of ISBSG have been considered along with the implications of the study for research and practice. Furthermore, its potential to develop new research in software engineering has also been outlined.

In the future, the authors intend to expand the period of collection of papers to keep the work up-to-date and to answer other interesting questions concerning the trend of usage of estimation methods, the potential relationship between estimation methods that could be 
combined, to identify the most relevant researchers and research groups that have used ISBSG, and to determine what are the most used variables in this type of research.

\section{Appendix A}

This section provides the primary studies selected from the mapping, sorted alphabetically:

[S1] S.-J. Huang, N.-H. Chiu, Y.-J. Liu, A comparative evaluation on the accuracies of software effort estimates from clustered data, Inf. Softw. Technol. 50 (2008) 879-888.

[S2] W. Zhang, Y. Yang, Q. Wang, A Comparative Study of Absent Features and Unobserved Values in Software Effort Data, Int. J. Softw. Eng. Knowl. Eng. 22 (2012) 185202.

[S3] J. Li, G. Ruhe, A comparative study of attribute weighting heuristics for effort estimation by analogy, in: Proceedings of the 2006 ACM/IEEE International Symposium on Empirical Software Engineering, ACM, New York, NY, USA, 2006: pp. 66-74.

[S4] R. Jeffery, M. Ruhe, I. Wieczorek, A comparative study of two software development cost modeling techniques using multi-organizational and company-specific data, Inf. Softw. Technol. 42 (2000) 1009-1016.

[S5] J. Li, G. Ruhe, A. Al-Emran, M.M. Richter, A flexible method for software effort estimation by analogy, Empir Software Eng. 12 (2007) 65-106.

[S6] X. Wang, Y. Zhang, L. Zhang, Y. Shi, A Knowledge Discovery Case Study of Software Quality Prediction: ISBSG Database, in: Web Intelligence and Intelligent Agent Technology (WI-IAT), 2010 IEEE/WIC/ACM International Conference on, 2010: pp. 219 222.

[S7] W. Xia, L.F. Capretz, D. Ho, F. Ahmed, A new calibration for Function Point complexity weights, Inf. Softw. Technol. 50 (2008) 670-683.

[S8] Q. Song, M. Shepperd, A new imputation method for small software project data sets, Journal of Systems and Software. 80 (2007) 51-62.

[S9] A. Bakır, B. Turhan, A.B. Bener, A new perspective on data homogeneity in software cost estimation: a study in the embedded systems domain, Software Qual J. 18 (2010) 57-80.

[S10] O. Adalier, A. Ugur, S. Korukoglu, K. Ertas, A new regression based software cost estimation model using power values, in: H. Yin, P. Tino, E. Corchado, W. Byrne, X. Yao (Eds.), Intelligent Data Engineering and Automated Learning - Ideal 2007, Springer-Verlag Berlin, Birmingham, ENGLAND, 2007: pp. 326-334.

[S11] N. Mittas, L. Angelis, A permutation test based on regression error characteristic curves for software cost estimation models, Empir Software Eng. 17 (2012) 34-61.

[S12] L.L. Minku, X. Yao, A principled evaluation of ensembles of learning machines for software effort estimation, in: Proceedings of the 7th International Conference on

Predictive Models in Software Engineering, ACM, New York, NY, USA, 2011: pp. 9:1-9:10. [S13] C. Symons, A process to explore the software project effort/duration trade-off relationship, Software, IEEE. (2011).

[S14] J. Moses, M. Farrow, N. Parrington, P. Smith, A productivity benchmarking case study using Bayesian credible intervals, Software Qual J. 14 (2006) 37-52. 
[S15] E. Mendes, C. Lokan, R. Harrison, C. Triggs, A replicated comparison of crosscompany and within-company effort estimation models using the ISBSG database, in: Software Metrics, 2005. 11th IEEE International Symposium, 2005: pp. $1-10$.

[S16] Q. Song, M. Shepperd, M. Cartwright, A Short Note on Safest Default Missingness Mechanism Assumptions, Empir Software Eng. 10 (2005) 235-243.

[S17] P. Sentas, L. Angelis, I. Stamelos, A statistical framework for analyzing the duration of software projects, Empir Software Eng. 13 (2008) 147-184.

[S18] V.K.Y. Chan, W.E. Wong, T.F. Xie, A Statistical Methodology to Simplify Software Metric Models Constructed Using Incomplete Data Samples, International Journal of Software Engineering and Knowledge Engineering. 17 (2007) 689-707.

[S19] Y.F. Li, M. Xie, T.N. Goh, A study of the non-linear adjustment for analogy based software cost estimation, Empir Software Eng. 14 (2009) 603-643.

[S20] E. Kocaguneli, A. Tosun, A. Bener, Al-Based Models for Software Effort Estimation, in: Software Engineering and Advanced Applications (SEAA), 2010 36th EUROMICRO Conference on, 2010: pp. $323-326$.

[S21] J.J. Cuadrado-Gallego, M.-A. Sicilia, An algorithm for the generation of segmented parametric software estimation models and its empirical evaluation, Comput. Inform. 26 (2007) $1-15$.

[S22] M. Hericko, A. Zivkovic, I. Roman, An approach to optimizing software development team size, Inf. Process. Lett. 108 (2008) 101-106.

[S23] C.J. Lokan, An empirical analysis of function point adjustment factors, Information and Software Technology. 42 (2000) 649-659.

[S24] Y.-S. Seo, K.-A. Yoon, D.-H. Bae, An empirical analysis of software effort estimation with outlier elimination, in: Proceedings of the 4th International Workshop on Predictor Models in Software Engineering, ACM, New York, NY, USA, 2008: pp. 25-32. [S25] M. Tsunoda, T. Kakimoto, A. Monden, K. Matsumoto, An empirical evaluation of outlier deletion methods for analogy-based cost estimation, in: Proceedings of the 7th International Conference on Predictive Models in Software Engineering, ACM, New York, NY, USA, 2011: pp. 17:1-17:10.

[S26] J.J. Cuadrado-Gallego, M.A. Sicilia, M. Garre, D. Rodriguez, An empirical study of process-related attributes in segmented software cost-estimation relationships, J. Syst. Softw. 79 (2006) 353-361.

[S27] P.C. Pendharkar, J.A. Rodger, G.H. Subramanian, An empirical study of the Cobb-Douglas production function properties of software development effort, Information and Software Technology. 50 (2008) 1181-1188.

[S28] K.K. Aggarwal, Y. Singh, P. Chandra, M. Puri, An expert committee model to estimate lines of code, SIGSOFT Softw. Eng. Notes. 30 (2005) 1-4.

[S29] T. Xie, W.E. Wong, An improved method to simplify software metric models constructed with incomplete data samples, in: Fuzzy Systems and Knowledge Discovery (FSKD), 2010 Seventh International Conference on, 2010: pp. 1682 -1688.

[S30] M. Azzeh, D. Neagu, P.I. Cowling, Analogy-based software effort estimation using Fuzzy numbers, J. Syst. Softw. 84 (2011) 270-284.

[S31] J. Li, G. Ruhe, Analysis of attribute weighting heuristics for analogy-based software effort estimation method AQUA+, Empir Software Eng. 13 (2008) 63-96. 
[S32] T. San Feliu, J.A. Calvo-Manzano, J.D. Carrillo, L.F. Mingo, Analysis of the International Software Benchmarking Standards Group (ISBSG) repository using Neural Nets, in: N. Callaos, E.S. ElBadawy, P. Conrad (Eds.), 6th World Multi-Conference on Systemics, Cybernetics and Informatics (SCI 2002)/8th International Conference on Information Systems Analysis and Synthesis (ISAS 2002), Int Inst Informatics \& Systemics, Orlando, 2002: pp. 461-466.

[S33] V.K.Y. Chan, W.E. Wong, T.F. Xie, Application of a Statistical Methodology to Simplify Software Quality Metric Models Constructed Using Incomplete Data Samples, in: Quality Software, 2006. QSIC 2006. Sixth International Conference on, 2006: pp. 15 -21. [S34] C. Lokan, E. Mendes, Applying moving windows to software effort estimation, in: Proceedings of the 2009 3rd International Symposium on Empirical Software Engineering and Measurement, IEEE Computer Society, Washington, DC, USA, 2009: pp. 111-122. [S35] W.E. Wong, J. Zhao, V.K.Y. Chan, Applying statistical methodology to optimize and simplify software metric models with missing data, in: Proceedings of the 2006 ACM Symposium on Applied Computing, ACM, New York, NY, USA, 2006: pp. 1728-1733. [S36] J. Moses, M. Farrow, Assessing Variation in Development Effort Consistency Using a Data Source with Missing Data, Software Qual J. 13 (2005) 71-89.

[S37] A.A. Issa, A.I. AlAli, Automated requirements engineering: Use case patternsdriven approach, Software, IET. 5 (2011) 287 -303.

[S38] A. Živkovič, I. Rozman, M. Heričko, Automated software size estimation based on function points using UML models, Information and Software Technology. 47 (2005) 881890.

[S39] S. Bibi, I. Stamelos, G. Gerolimos, V. Kollias, BBN based approach for improving the software development process of an SME - a case study, J. Softw. Maint. Evol.-Res. Pract. 22 (2010) 121-140.

[S40] L. Angelis, I. Stamelos, M. Morisio, Building a software cost estimation model based on categorical data, in: Software Metrics Symposium, 2001. METRICS 2001. Proceedings. Seventh International, 2001: pp. 4-15.

[S41] Q. Song, M. Shepperd, X. Chen, J. Liu, Can k-NN imputation improve the performance of $\mathrm{C} 4.5$ with small software project data sets? A comparative evaluation, Journal of Systems and Software. 81 (2008) 2361-2370.

[S42] P. Sentas, L. Angelis, Categorical missing data imputation for software cost estimation by multinomial logistic regression, J. Syst. Softw. 79 (2006) 404-414.

[S43] S. Bibi, I. Stamelos, L. Angelis, Combining probabilistic models for explanatory productivity estimation, Information and Software Technology. 50 (2008) 656-669.

[S44] N. Mittas, L. Angelis, Combining regression and estimation by analogy in a semiparametric model for software cost estimation, in: Proceedings of the Second ACM-IEEE International Symposium on Empirical Software Engineering and Measurement, ACM, New York, NY, USA, 2008: pp. 70-79.

[S45] N. Mittas, L. Angelis, Comparing cost prediction models by resampling techniques, Journal of Systems and Software. 81 (2008) 616-632.

[S46] N. Mittas, L. Angelis, Comparing Software Cost Prediction Models by a Visualization Tool, in: Software Engineering and Advanced Applications, 2008. SEAA '08. 34th Euromicro Conference, 2008: pp. 433 -440. 
[S47] S. Berlin, T. Raz, C. Glezer, M. Zviran, Comparison of estimation methods of cost and duration in IT projects, Information and Software Technology. 51 (2009) 738-748.

[S48] B. Twala, M. Cartwright, M. Shepperd, Comparison of various methods for handling incomplete data in software engineering databases, in: Empirical Software Engineering, 2005. 2005 International Symposium on, 2005: pp. $105-114$.

[S49] C.-J. Hsu, C.-Y. Huang, Comparison of weighted grey relational analysis for software effort estimation, Software Qual J. 19 (2011) 165-200.

[S50] C. Lokan, E. Mendes, Cross-company and single-company effort models using the ISBSG database: a further replicated study, in: Proceedings of the $2006 \mathrm{ACM} / \mathrm{IEEE}$ International Symposium on Empirical Software Engineering, ACM, New York, NY, USA, 2006: pp. 75-84.

[S51] K. Dejaeger, W. Verbeke, D. Martens, B. Baesens, Data Mining Techniques for Software Effort Estimation: A Comparative Study, Software Engineering, IEEE Transactions on. 38 (2012) $375-397$.

[S52] P. Bourque, S. Oligny, A. Abran, B. Fournier, Developing project duration models in software engineering, J. Comput. Sci. Technol. 22 (2007) 348-357.

[S53] C. Gencel, L. Buglione, Do Base Functional Component types affect the relationship between software functional size and effort?, in: J.J. CuadradoGallego, R. Braungarten, R.R. Dumke, A. Abran (Eds.), Software Process and Product Measurement, Springer-Verlag Berlin, Palma de Majorque, SPAIN, 2008: pp. 72-85.

[S54] Y. Kultur, E. Kocaguneli, A.B. Bener, Domain specific phase by phase effort estimation in software projects, in: Computer and Information Sciences, 2009. ISCIS 2009. 24th International Symposium on, 2009: pp. 498 -503.

[S55] C. Comstock, Z. Jiang, J. Davies, Economies and diseconomies of scale in software development, Journal of Software Maintenance and Evolution: Research and Practice. 23 (2011) 533-548.

[S56] M. Castejon-Limas, J. Ordieres-Mere, A. Gonzalez-Marcos, V. Gonzalez-Castro, Effort estimates through project complexity, Ann. Oper. Res. 186 (2011) 395-406.

[S57] J. Aziz, F. Ahmed, M.S. Laghari, Empirical Analysis of Team and Application Size on Software Maintenance and Support Activities, in: Information Management and Engineering, 2009. ICIME '09. International Conference on, 2009: pp. 47 -51.

[S58] D. Rodriguez, M.A. Sicilia, E. Garcia, R. Harrison, Empirical findings on team size and productivity in software development, J. Syst. Softw. 85 (2012) 562-570.

[S59] B. Twala, M. Cartwright, Ensemble imputation methods for missing software engineering data, in: Software Metrics, 2005. 11th IEEE International Symposium, 2005:

pp. $1-10$.

[S60] G. Sikka, A. Kaur, M. Uddin, Estimating function points: Using machine learning and regression models, in: Education Technology and Computer (ICETC), 2010 2nd International Conference on, 2010: pp. $52-56$.

[S61] I. Stamelos, L. Angelis, M. Morisio, E. Sakellaris, G.L. Bleris, Estimating the development cost of custom software, Information \& Management. 40 (2003) 729-741. [S62] Q. Liu, W.Z. Qin, R. Mintram, M. Ross, Evaluation of preliminary data analysis framework in software cost estimation based on ISBSG R9 Data, Softw. Qual. J. 16 (2008) 411-458. 
[S63] K.K. Aggarwal, Y. Singh, P. Chandra, M. Puri, Evaluation of various training algorithms in a neural network model for software engineering applications, SIGSOFT Softw. Eng. Notes. 30 (2005) 1-4.

[S64] A.S. Andreou, E. Papatheocharous, C. Skouroumounis, Evolving Conditional Value Sets of Cost Factors for Estimating Software Development Effort, in: Tools with Artificial Intelligence, 2007. ICTAI 2007. 19th IEEE International Conference on, 2007: pp. $165-172$.

[S65] J. Keung, B. Kitchenham, Experiments with Analogy-X for Software Cost Estimation, in: Software Engineering, 2008. ASWEC 2008. 19th Australian Conference on, 2008: pp. $229-238$.

[S66] E. Kocaguneli, T. Menzies, A. Bener, J.W. Keung, Exploiting the Essential Assumptions of Analogy-Based Effort Estimation, Software Engineering, IEEE Transactions on. 38 (2012) $425-438$.

[S67] E.N. Regolin, G.A. de Souza, A.R.T. Pozo, S.R. Vergilio, Exploring machine learning techniques for software size estimation, in: Chilean Computer Science Society, 2003. SCCC 2003. Proceedings. 23rd International Conference of the, 2003: pp. 130 136.

[S68] T.K. Le-Do, K.-A. Yoon, Y.-S. Seo, D.-H. Bae, Filtering of Inconsistent Software Project Data for Analogy-Based Effort Estimation, in: Computer Software and Applications Conference (COMPSAC), 2010 IEEE 34th Annual, 2010: pp. 503 -508.

[S69] K. Toda, A. Monden, K. Matsumoto, Fit data selection for software effort estimation models, in: Proceedings of the Second ACM-IEEE International Symposium on Empirical Software Engineering and Measurement, ACM, New York, NY, USA, 2008: pp. 360-361.

[S70] C. Gencel, O. Demirors, Functional size measurement revisited, ACM Trans. Softw. Eng. Methodol. 17 (2008) 15:1-15:36.

[S71] M. Azzeh, D. Neagu, P.I. Cowling, Fuzzy grey relational analysis for software effort estimation, Empir Software Eng. 15 (2010) 60-90.

[S72] W. Zhang, Y. Yang, Q. Wang, Handling missing data in software effort prediction with naive Bayes and EM algorithm, in: Proceedings of the 7th International Conference on Predictive Models in Software Engineering, ACM, New York, NY, USA, 2011: pp. 4:1-4:10. [S73] J. Li, A. Al-Emran, G. Ruhe, Impact Analysis of Missing Values on the Prediction Accuracy of Analogy-based Software Effort Estimation Method AQUA, in: Empirical Software Engineering and Measurement, 2007. ESEM 2007. First International Symposium on, 2007: pp. $126-135$.

[S74] L. Buglione, C. Gencel, Impact of base functional component types on software functional size based effort estimation, in: A. Jedlitschka, O. Salo (Eds.), Product-Focused Software Process Improvement, Proceedings, Frascati, ITALY, 2008: pp. 75-89.

[S75] T. Kralj, I. Rozman, M. Hericko, A. Zivkovic, Improved standard FPA methodresolving problems with upper boundaries in the rating complexity process, J. Syst. Softw. 77 (2005) 81-90.

[S76] M. Azzeh, D. Neagu, P. Cowling, Improving analogy software effort estimation using fuzzy feature subset selection algorithm, in: Proceedings of the 4th International Workshop on Predictor Models in Software Engineering, ACM, New York, NY, USA, 2008: pp. 71-78. 
[S77] C.-J. Hsu, C.-Y. Huang, Improving Effort Estimation Accuracy by Weighted Grey Relational Analysis During Software Development, in: Software Engineering Conference, 2007. APSEC 2007. 14th Asia-Pacific, 2007: pp. $534-541$.

[S78] Y.-S. Seo, K.-A. Yoon, D.-H. Bae, Improving the Accuracy of Software Effort Estimation Based on Multiple Least Square Regression Models by Estimation Error-Based Data Partitioning, in: Software Engineering Conference, 2009. APSEC '09. Asia-Pacific, 2009: pp. $3-10$.

[S79] F. Ahmed, S. Bouktif, A. Serhani, I. Khalil, Integrating Function Point Project Information for Improving the Accuracy of Effort Estimation, in: Advanced Engineering Computing and Applications in Sciences, 2008. ADVCOMP '08. The Second International Conference on, 2008: pp. $193-198$.

[S80] O.O. Top, B. Ozkan, M. Nabi, O. Demirors, Internal and External Software Benchmark Repository Utilization for Effort Estimation, in: Software Measurement, 2011 Joint Conference of the 21st Int'I Workshop on and 6th Int'I Conference on Software Process and Product Measurement (IWSM-MENSURA), 2011: pp. 302 -307.

[S81] C. Lokan, E. Mendes, Investigating the use of chronological split for software effort estimation, Software, IET. 3 (2009) $422-434$.

[S82] B.L. Dalmazo, W.L. Cordeiro, L. Rabelo, J.A. Wickboldt, R.C. Lunardi, R.L. dos Santos, et al., Leveraging IT project lifecycle data to predict support costs, in: Integrated Network Management (IM), 2011 IFIP/IEEE International Symposium on, 2011: pp. 249 256.

[S83] N. Mittas, L. Angelis, LSEbA: least squares regression and estimation by analogy in a semi-parametric model for software cost estimation, Empir Software Eng. 15 (2010) 523-555.

[S84] M. Azzeh, Model Tree Based Adaption Strategy for Software Effort Estimation by Analogy, in: Computer and Information Technology (CIT), 2011 IEEE 11th International Conference on, 2011: pp. $328-335$.

[S85] M.A. Al-Hajri, A.A. Abdul Ghani, M.N. Sulaiman, M.H. Selamat, Modification of standard Function Point complexity weights system, Journal of Systems and Software. 74 (2005) 195-206.

[S86] J. Li, G. Ruhe, Multi-criteria decision analysis for customization of estimation by analogy method AQUA+, in: Proceedings of the 4th International Workshop on Predictor Models in Software Engineering, ACM, New York, NY, USA, 2008: pp. 55-62.

[S87] K. Haaland, I. Stamelos, R. Ghosh, R. Glott, On the Approximation of the Substitution Costs for Free/Libre Open Source Software, in: Informatics, 2009. BCl '09. Fourth Balkan Conference in, 2009: pp. 223 -227.

[S88] C. Gencel, R. Heldal, K. Lind, On the Relationship between Different Size Measures in the Software Life Cycle, in: Software Engineering Conference, 2009. APSEC '09. Asia-Pacific, 2009: pp. 19 -26.

[S89] M. llyas, J. Kung, Ontology-Based Similarity Measurement in Software Projects through SimReq Framework, in: Software Engineering Advances (ICSEA), 2010 Fifth International Conference on, 2010: pp. $412-416$.

[S90] S.-J. Huang, N.-H. Chiu, Optimization of analogy weights by genetic algorithm for software effort estimation, Inf. Softw. Technol. 48 (2006) 1034-1045. 
[S91] V.K.Y. Chan, W.E. Wong, Optimizing and simplifying software metric models constructed using maximum likelihood methods, in: Computer Software and Applications Conference, 2005. COMPSAC 2005. 29th Annual International, 2005: pp. 1 -6.

[S92] C. Lokan, T. Wright, P. Hill, M. Stringer, Organizational benchmarking using the ISBSG Data Repository, Software, IEEE. 18 (2001) 26 -32.

[S93] V.K.Y. Chan, W.E. Wong, Outlier elimination in construction of software metric models, in: Proceedings of the 2007 ACM Symposium on Applied Computing, ACM, New York, NY, USA, 2007: pp. 1484-1488.

[S94] B. Stewart, Predicting project delivery rates using the Naive-Bayes classifier, Journal of Software Maintenance and Evolution: Research and Practice. 14 (2002) 161179.

[S95] Q. Liu, R. Mintram, Preliminary data analysis methods in software estimation, Softw. Qual. J. 13 (2005) 91-115.

[S96] J.L. Eveleens, M. van der Pas, C. Verhoef, Quantifying forecast quality of IT business value, Science of Computer Programming. 77 (2012) 314-354.

[S97] R. Abdukalykov, I. Hussain, M. Kassab, O. Ormandjieva, Quantifying the Impact of Different Non-functional Requirements and Problem Domains on Software Effort Estimation, in: Software Engineering Research, Management and Applications (SERA), 2011 9th International Conference on, 2011: pp. 158 -165.

[S98] S. Bibi, G. Tsoumakas, I. Stamelos, I. Vlahavas, Regression via Classification applied on software defect estimation, Expert Syst. Appl. 34 (2008) 2091-2101.

[S99] E. Mendes, C. Lokan, Replicating studies on cross- vs single-company effort models using the ISBSG Database, Empir. Softw. Eng. 13 (2008) 3-37.

[S100] A.A. Issa, M. Al-Diabat, Reverse engineering Function Points to Object Points, in: Applications of Digital Information and Web Technologies, 2009. ICADIWT '09. Second International Conference on the, 2009: pp. $151-156$.

[S101] D. Rodríguez, J.J. Cuadrado, M.A. Sicilia, R. Ruiz, Segmentation of software engineering datasets using the $\mathrm{m} 5$ algorithm, in: Proceedings of the 6th International Conference on Computational Science - Volume Part IV, Springer-Verlag, Berlin, Heidelberg, 2006: pp. 789-796.

[S102] M. Garre, J.J. Cuadrado, M.A. Sicilia, M. Charro, D. Rodriguez, Segmented parametric software estimation models: using the EM algorithm with the ISBSG 8 database, in: Information Technology Interfaces, 2005. 27th International Conference on, 2005: pp. $181-187$.

[S103] J. Aroba, J.J. Cuadrado-Gallego, M.-A. Sicilia, I. Ramos, E. Garcia-Barriocanal, Segmented software cost estimation models based on fuzzy clustering, J. Syst. Softw. 81 (2008) 1944-1950.

[S104] S.J. Huang, W.M. Han, Selection priority of process areas based on CMMI continuous representation, Inf. Manage. 43 (2006) 297-307.

[S105] M. Fernández-Diego, M. Martínez-Gómez, J.-M. Torralba-Martínez, Sensitivity of results to different data quality meta-data criteria in the sample selection of projects from the ISBSG dataset, in: Proceedings of the 6th International Conference on Predictive Models in Software Engineering, ACM, New York, NY, USA, 2010: pp. 13:1-13:9. 
[S106] T. Xie, W.E. Wong, W. Ding, Simplifying Software Metric Models via Hierarchical LASSO with Incomplete Data Samples, in: Software Engineering (WCSE), 2010 Second World Congress on, 2010: pp. $161-164$.

[S107] E. Papatheocharous, A.S. Andreou, Software cost estimation using artificial neural networks with inputs selection, in: J. Cardoso, J. Cordoso, J. Filipe (Eds.), Insticc-Inst Syst Technologies Information Control \& Communication, Funchal, PORTUGAL, 2007: pp. 398-407.

[S108] J.S. Pahariya, V. Ravi, M. Carr, Software cost estimation using computational intelligence techniques, in: Nature Biologically Inspired Computing, 2009. NaBIC 2009. World Congress on, 2009: pp. 849 -854.

[S109] A.S. Andreou, E. Papatheocharous, Software Cost Estimation using Fuzzy Decision Trees, in: Proceedings of the 2008 23rd IEEE/ACM International Conference on Automated Software Engineering, IEEE Computer Society, Washington, DC, USA, 2008: pp. 371-374.

[S110] A. Issa, M. Odeh, D. Coward, Software Cost Estimation using Use-Case Models: a Critical Evaluation, in: Information and Communication Technologies, 2006. ICTTA '06. 2nd, 2006: pp. $2766-2771$.

[S111] M. Azzeh, Software effort estimation based on optimized model tree, in: Proceedings of the 7th International Conference on Predictive Models in Software Engineering, ACM, New York, NY, USA, 2011: pp. 6:1-6:8.

[S112] J. Li, G. Ruhe, Software Effort Estimation by Analogy Using Attribute Selection Based on Rough Set Analysis, International Journal of Software Engineering and Knowledge Engineering. 18 (2008) 1-23.

[S113] R. Setiono, K. Dejaeger, W. Verbeke, D. Martens, B. Baesens, Software Effort Prediction Using Regression Rule Extraction from Neural Networks, in: Tools with Artificial Intelligence (ICTAI), 2010 22nd IEEE International Conference on, 2010: pp. 45 -52. [S114] P. Sentas, L. Angelis, I. Stamelos, G. Bleris, Software productivity and effort prediction with ordinal regression, Information and Software Technology. 47 (2005) 17-29. [S115] J.J. Cuadrado Gallego, D. Rodriguez, M. Angel Sicilia, M.G. Rubio Angel, A.G. Crespo, Software project effort estimation based on multiple parametric models generated through data clustering, J. Comput. Sci. Technol. 22 (2007) 371-378.

[S116] Y. Shan, R.I. McKay, C.J. Lokan, D.L. Essam, Software project effort estimation using genetic programming, in: Communications, Circuits and Systems and West Sino Expositions, IEEE 2002 International Conference on, 2002: pp. 1108 - 1112 vol.2.

[S117] M. Azzeh, P.I. Cowling, D. Neagu, Software Stage-Effort Estimation Based on Association Rule Mining and Fuzzy Set Theory, in: Computer and Information Technology (CIT), 2010 IEEE 10th International Conference on, 2010: pp. 249 -256.

[S118] P. Sentas, L. Angelis, Survival analysis for the duration of software projects, in: Software Metrics, 2005. 11th IEEE International Symposium, 2005: pp. 1 -10.

[S119] Z. Jiang, C. Comstock, The Factors Significant to Software Development Productivity, in: C. Ardil (Ed.), Proceedings of World Academy of Science, Engineering and Technology, Vol 19, World Acad Sci, Eng \& Tech-Waset, Bangkok, THAILAND, 2007: pp. 160-164.

[S120] P.C. Pendharkar, J.A. Rodger, The relationship between software development team size and software development cost, Commun. ACM. 52 (2009) 141-144. 
[S121] Y. Zorgios, O. Vlismas, G. Venieris, The SECI Model and the Learning Curve Phenomenon, in: C. Stam (Ed.), PROCEEDINGS OF THE EUROPEAN CONFERENCE ON INTELLECTUAL CAPITAL, Academic Conferences Ltd, INHolland Univ Appl Sci, Haarlem, NETHERLANDS, 2009: pp. 589-599.

[S122] Z. Jiang, P. Naude, C. Comstock, The Variation of Software Development Productivity 1995-2005, in: C. Ardil (Ed.), Proceedings of World Academy of Science, Engineering and Technology, Vol 21, World Acad Sci, Eng \& Tech-Waset, Vienna, AUSTRIA, 2007: pp. 355-359.

[S123] C. Lokan, E. Mendes, Using chronological splitting to compare cross- and singlecompany effort models: further investigation, in: Proceedings of the Thirty-Second Australasian Conference on Computer Science - Volume 91, Australian Computer Society, Inc., Darlinghurst, Australia, Australia, 2009: pp. 47-54.

[S124] Q. Liu, R. Mintram, Using industry based data sets in software engineering research, in: Proceedings of the 2006 International Workshop on Summit on Software Engineering Education, ACM, New York, NY, USA, 2006: pp. 33-36.

[S125] R. Jeffery, M. Ruhe, I. Wieczorek, Using public domain metrics to estimate software development effort, in: Software Metrics Symposium, 2001. METRICS 2001. Proceedings. Seventh International, 2001: pp. 16 -27.

[S126] A.A. Issa, F.A.A. Rub, F.F. Thabata, Using test case patterns to estimate software development and quality management cost, Software Qual J. 17 (2009) 263-281.

[S127] N. Mittas, L. Angelis, Visual comparison of software cost estimation models by regression error characteristic analysis, Journal of Systems and Software. 83 (2010) 621637.

[S128] S.S. Bajwa, C. Gencel, What Are the Significant Cost Drivers for COSMIC Functional Size Based Effort Estimation?, in: A. Abran, R. Braungarten, R.R. Dumke, J.J. Cuadrado Gallego, J. Brunekreef (Eds.), Software Process and Product Measurement, Proceedings, Springer-Verlag Berlin, Hogesch van Amsterdam, Amsterdam, NETHERLANDS, 2009: pp. 62-75.

[S129] C. Lokan, What should you optimize when building an estimation model?, in: Software Metrics, 2005. 11th IEEE International Symposium, 2005: pp. 1-10.

\section{Bibliography}

[1] ISBSG, ISBSG dataset Release 12, Int. Softw. Benchmarking Stand. Group. (2013).

[2] E. Stensrud, T. Foss, B. Kitchenham, I. Myrtveit, An empirical validation of the relationship between the magnitude of relative error and project size, in: Softw. Metr. 2002 Proc. Eighth IEEE Symp. On, 2002: pp. 3-12.

[3] G.A. Liebchen, M. Shepperd, Data sets and data quality in software engineering, in: Proc. 4th Int. Workshop Predict. Models Softw. Eng. PROMISE, ACM, Leipzig, Germany, 2008: pp. 39-44.

[4] W. Afzal, R. Torkar, R. Feldt, A systematic mapping study on non-functional search-based software testing, in: Proc. 20th Int. Conf. Softw. Eng. Knowl. Eng. SEKE'08 Knowl. Syst. Inst. Grad. Sch., 2008.

[5] M. Petticrew, H. Roberts, Systematic Reviews In The Social Sciences: A Practical Guide, John Wiley \& Sons, Limited, 2006. 
[6] B. Kitchenham, S. Charters, Guidelines for performing Systematic Literature Reviews in Software Engineering, Sofwtare Engineering Group, School of Computer Science and Mathematics, Keele University, 2007.

[7] K. Petersen, R. Feldt, S. Mujtaba, M. Mattsson, Systematic mapping studies in software engineering, in: 12th Int. Conf. Eval. Assess. Softw. Eng., 2008: p. 1.

[8] D. Budgen, M. Turner, P. Brereton, B. Kitchenham, Using mapping studies in software engineering, in: Proc. PPIG, 2008: pp. 195-204.

[9] B.A. Kitchenham, D. Budgen, P. Brereton, The value of mapping studies-a participant-observer case study, in: Proc. 14th Int. Conf. Eval. Assess. Softw. Eng. EASE'10, 2010.

[10] S.T. Acuna, J.W. Castro, O. Dieste, N. Juristo, A systematic mapping study on the open source software development process, in: Eval. Assess. Softw. Eng. EASE 2012 16th Int. Conf. On, 2012: pp. 42-46.

[11] N. Asoudeh, Y. Labiche, Requirement-based Software Testing With the UML: A Systematic Mapping Study, in: ICSEA 2012 Seventh Int. Conf. Softw. Eng. Adv., 2012: pp. 623-629.

[12] H. Zhang, M.A. Babar, P. Tell, Identifying relevant studies in software engineering, Inf. Softw. Technol. 53 (2011) 625-637.

[13] F. Elberzhager, J. Münch, V.T.N. Nha, A systematic mapping study on the combination of static and dynamic quality assurance techniques, Inf. Softw. Technol. 54 (2012) 1-15.

[14] The Excellence in Research for Australia (ERA) Initiative, (n.d.).

[15] M. Jorgensen, M. Shepperd, A systematic review of software development cost estimation studies, Softw. Eng. IEEE Trans. On. 33 (2007) 33-53.

[16] Y. Yang, M. He, M. Li, Q. Wang, B. Boehm, Phase distribution of software development effort, in: Proc. Second ACM-IEEE Int. Symp. Empir. Softw. Eng. Meas., ACM, New York, NY, USA, 2008: pp. 61-69.

[17] K.D. Maxwell, Applied Statistics for Software Managers, Prentice Hall, 2002.

[18] M. Shepperd, C. Schofield, B. Poole, Effort Estimation Using Analogy, (1996).

[19] F. Walkerden, R. Jeffery, An Empirical Study of Analogy-based Software Effort Estimation, Empir. Softw Engg. 4 (1999) 135-158.

[20] M.A. Ahmed, Z. Muzaffar, Handling imprecision and uncertainty in software development effort prediction: A type-2 fuzzy logic based framework, Inf. Softw. Technol. 51 (2009) 640-654.

[21] Z. Muzaffar, M.A. Ahmed, Software development effort prediction: A study on the factors impacting the accuracy of fuzzy logic systems, Inf. Softw. Technol. 52 (2010) 92109.

[22] M. Bundschuh, C. Dekkers, The IT Measurement Compendium, Springer, 2008.

[23] C. Mair, M. Shepperd, M. Jørgensen, An analysis of data sets used to train and validate cost prediction systems, in: Proc. 2005 Workshop Predict. Models Softw. Eng., ACM, St. Louis, Missouri, 2005: pp. 1-6.

[24] J.M. Desharnais, Analyse Statistique de la Productivite des Projets de Developpement en Informatique a Partir de la Technique des Points de Fonction, maîtrise informatique de gestion, Univ. Quebec Montr. (1988).

[25] B.W. Boehm, Software Engineering Economics, Pearson Education, 1981.

[26] C.F. Kemerer, An empirical validation of software cost estimation models, Commun ACM. 30 (1987) 416-429.

[27] A.J. Albrecht, J.E. Gaffney Jr, Software function, source lines of code, and development effort prediction: a software science validation, Softw. Eng. IEEE Trans. On. (1983) 639-648.

[28] G. Boetticher, T. Menzies, T. Ostrand, PROMISE Repository of empirical software engineering data, W. Va. Univ. Dep. Comput. Sci. (2007). 
[29] E. Mendes, I. Watson, C. Triggs, N. Mosley, S. Counsell, A comparative study of cost estimation models for web hypermedia applications, Empir. Softw. Eng. 8 (2003) 163196.

[30] R. Premraj, M. Shepperd, B. Kitchenham, P. Forselius, An empirical analysis of software productivity over time, in: Softw. Metr. 2005 11th IEEE Int. Symp., 2005: p. 10.

[31] Y. Kultur, B. Turhan, A.B. Bener, ENNA: software effort estimation using ensemble of neural networks with associative memory, in: Proc. 16th ACM SIGSOFT Int. Symp.

Found. Softw. Eng., 2008: pp. 330-338.

[32] A. Abran, P.N. Robillard, Function points analysis: an empirical study of its measurement processes, IEEE Trans. Softw. Eng. 22 (1996) 895-910.

[33] M. He, M. Li, Q. Wang, Y. Yang, K. Ye, An investigation of software development productivity in China, in: Mak. Glob. Distrib. Softw. Dev. Success Story, Springer, 2008: pp. 381-394.

[34] C. Robson, Real World Research: A Resource for Social Scientists and Practitioner-Researchers, Wiley, 2002.

[35] B. Littlewood, P. Popov, L. Strigini, Modeling Software Design Diversity: A Review, ACM Comput Surv. 33 (2001) 177-208.

[36] Y.-S. Seo, D.-H. Bae, On the value of outlier elimination on software effort estimation research, Empir. Softw. Eng. 18 (2013) 659-698.

[37] T. Dyba, T. Dingsoyr, G.K. Hanssen, Applying systematic reviews to diverse study types: An experience report, in: Empir. Softw. Eng. Meas. 2007 ESEM 2007 First Int.

Symp. On, 2007: pp. 225-234. 


\section{Figure Captions}

Figure 1: Search process for the selection of studies.

Figure 2: Filtering process.

Figure 3: Number of papers in journals and conferences over time.

Figure 4: Number of papers published per year.

Figure 5: Number of identified and cited articles and received citations per cited article.

Figure 6: Further considered variables apart from effort.

Figure 7: Dataset properties.

Figure 8: Evolution over time of the three most frequent topics.

Figure 9: Evolution over time of the three most frequent estimation methods used.

Figure 10: Evolution over time of the three most frequently used estimation methods in journals with ISBSG-based research papers.

Figure 11: ISBSG releases and the evolution of the number of papers using a specific release of ISBSG.

(a): ISBSG releases over time.

(b): Evolution of the number of papers using a specific release of ISBSG.

\section{Table Captions}

Table 1: Data extraction form to collect information from each identified paper.

Table 2: Journal type publication sources of selected papers.

Table 3: Conference type sources with two or more selected papers.

Table 4: Top-cited identified papers with at least 6 citations.

Table 5: Citations received by identified papers in dataset journals over time.

Table 6: Number and proportion of papers for each research topic.

Table 7: Number and proportion of papers for each estimation method.

Table 8: Machine Learning estimation methods.

Table 9: Other datasets used apart from ISBSG. 
Figure 1: Search process for the selection of studies.

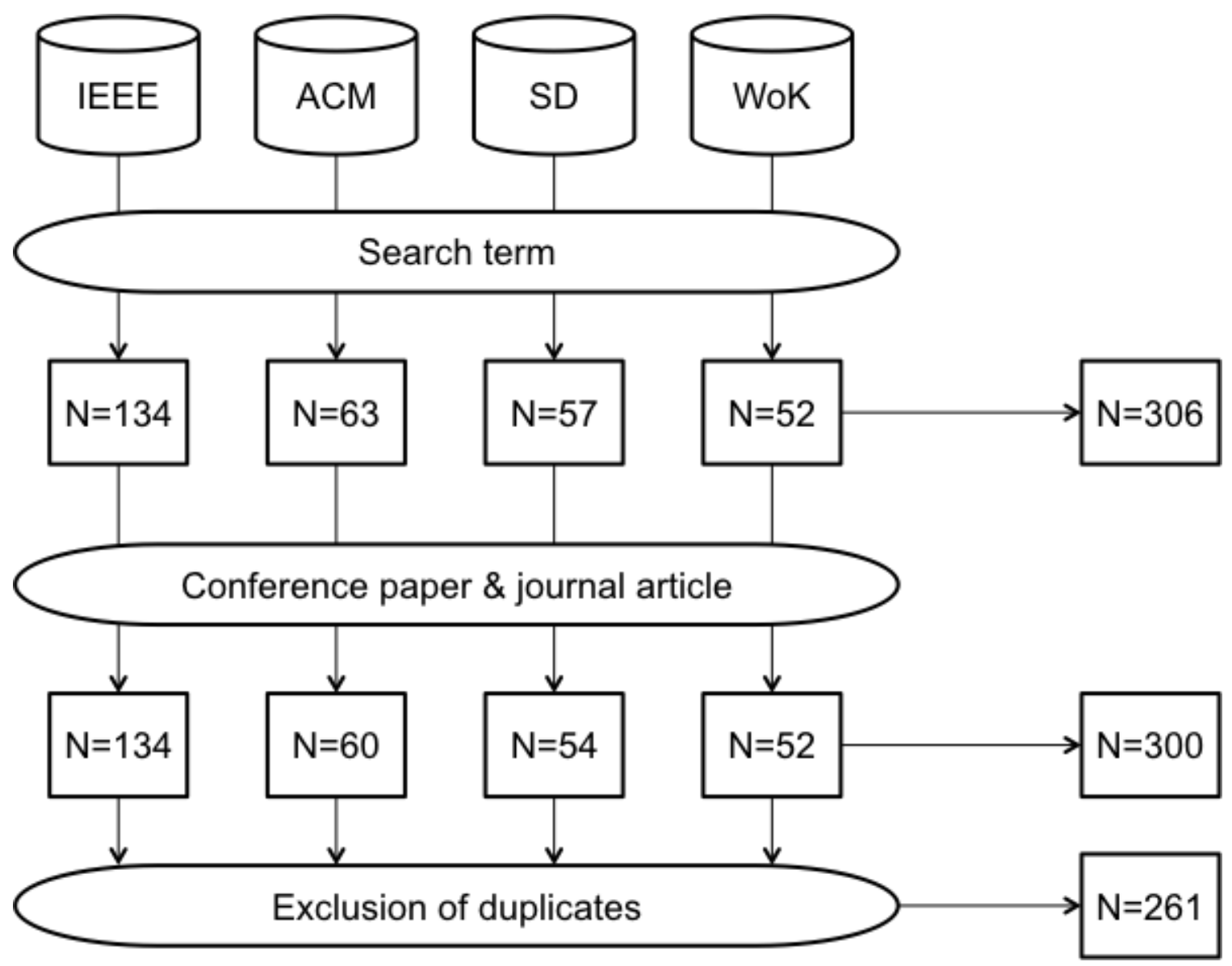


Figure 2: Filtering process.

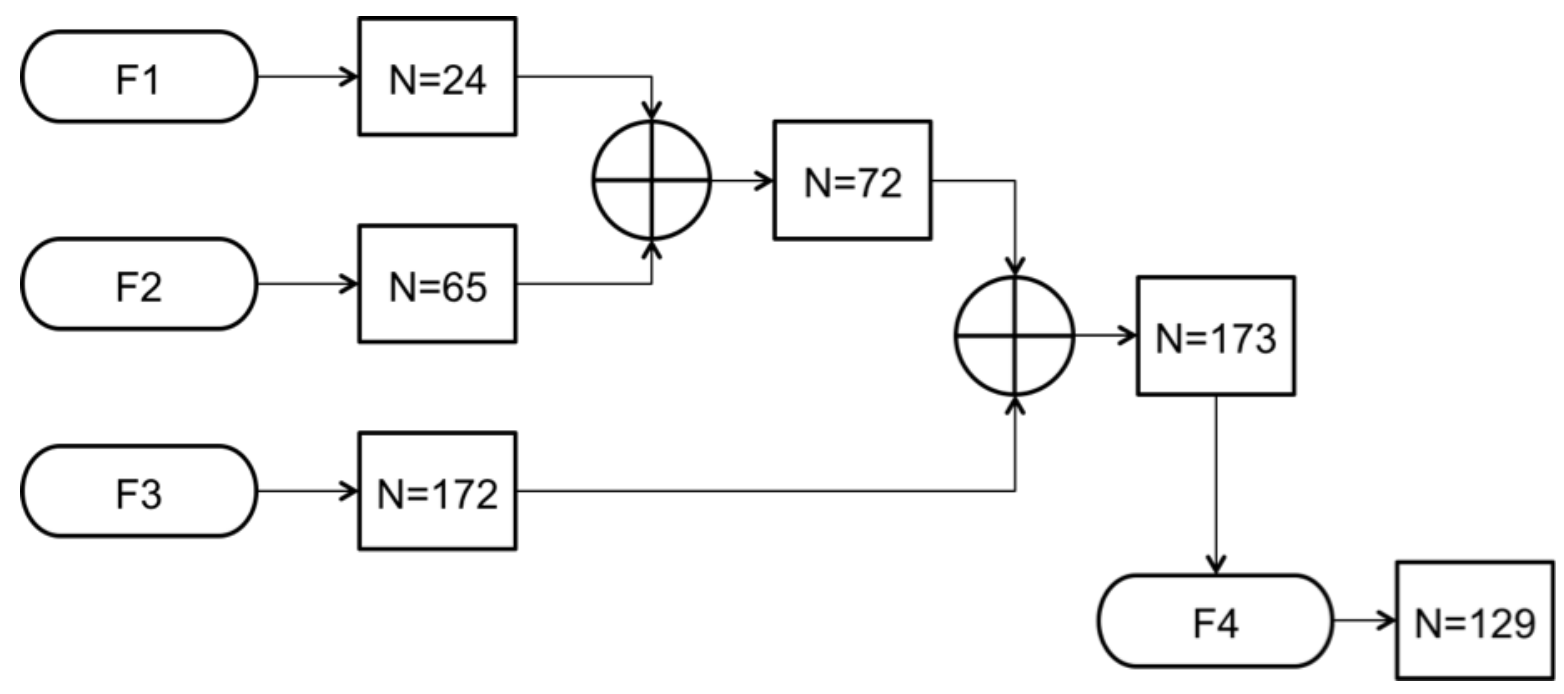


Figure 3: Number of papers in journals and conferences over time.

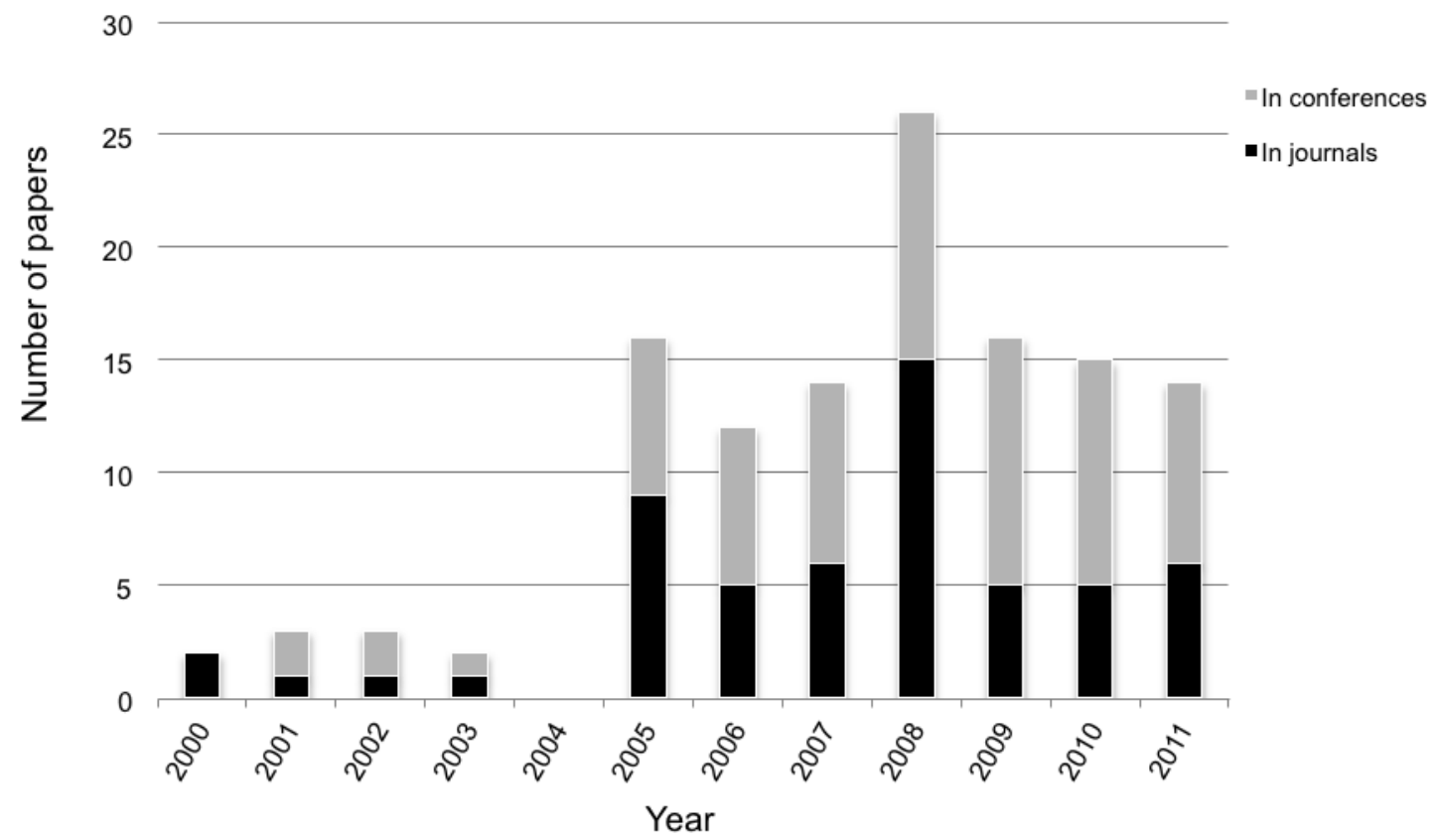


Figure 4: Number of papers published per year.

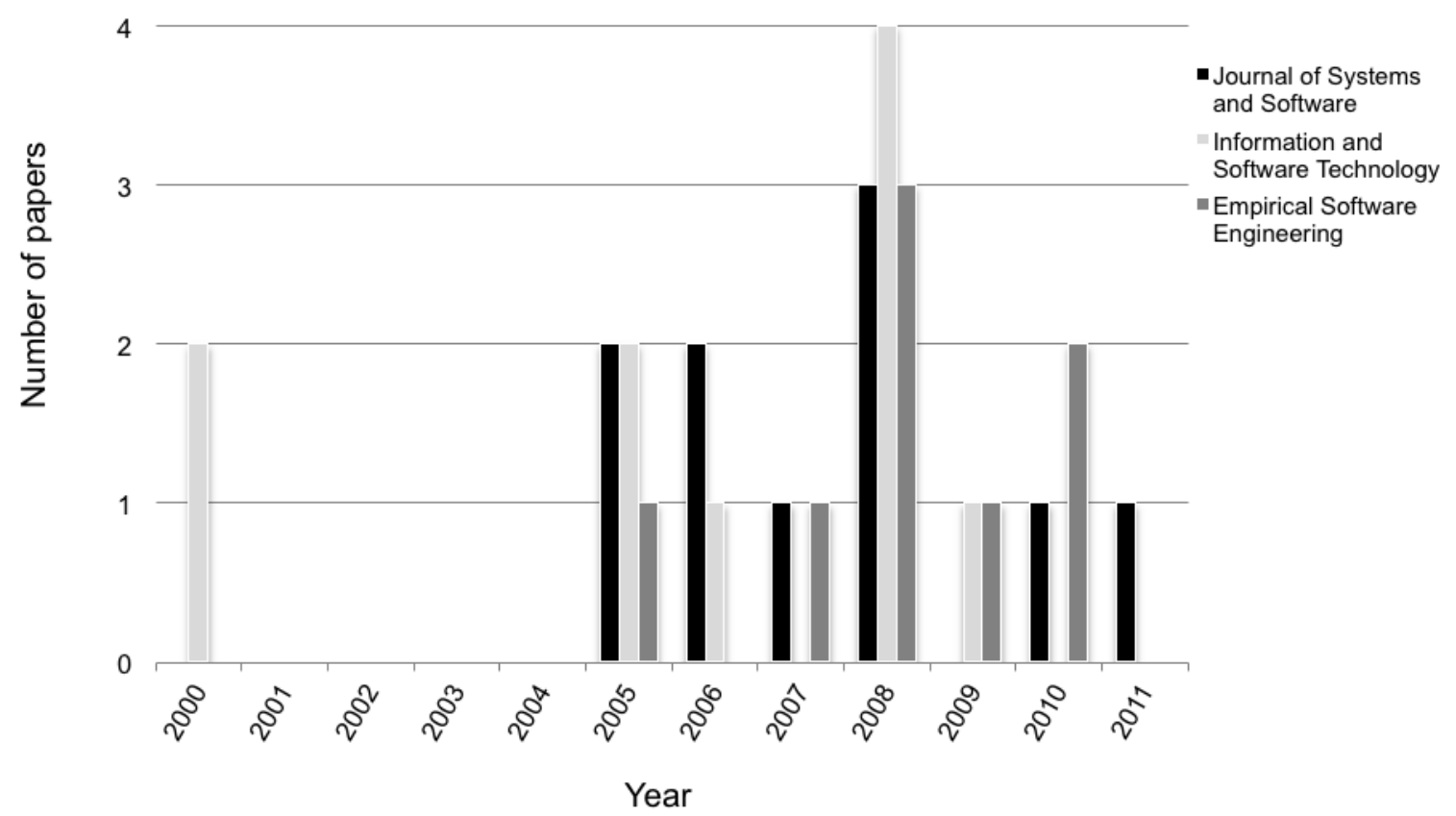


Figure 5: Number of identified and cited articles and received citations per cited article.

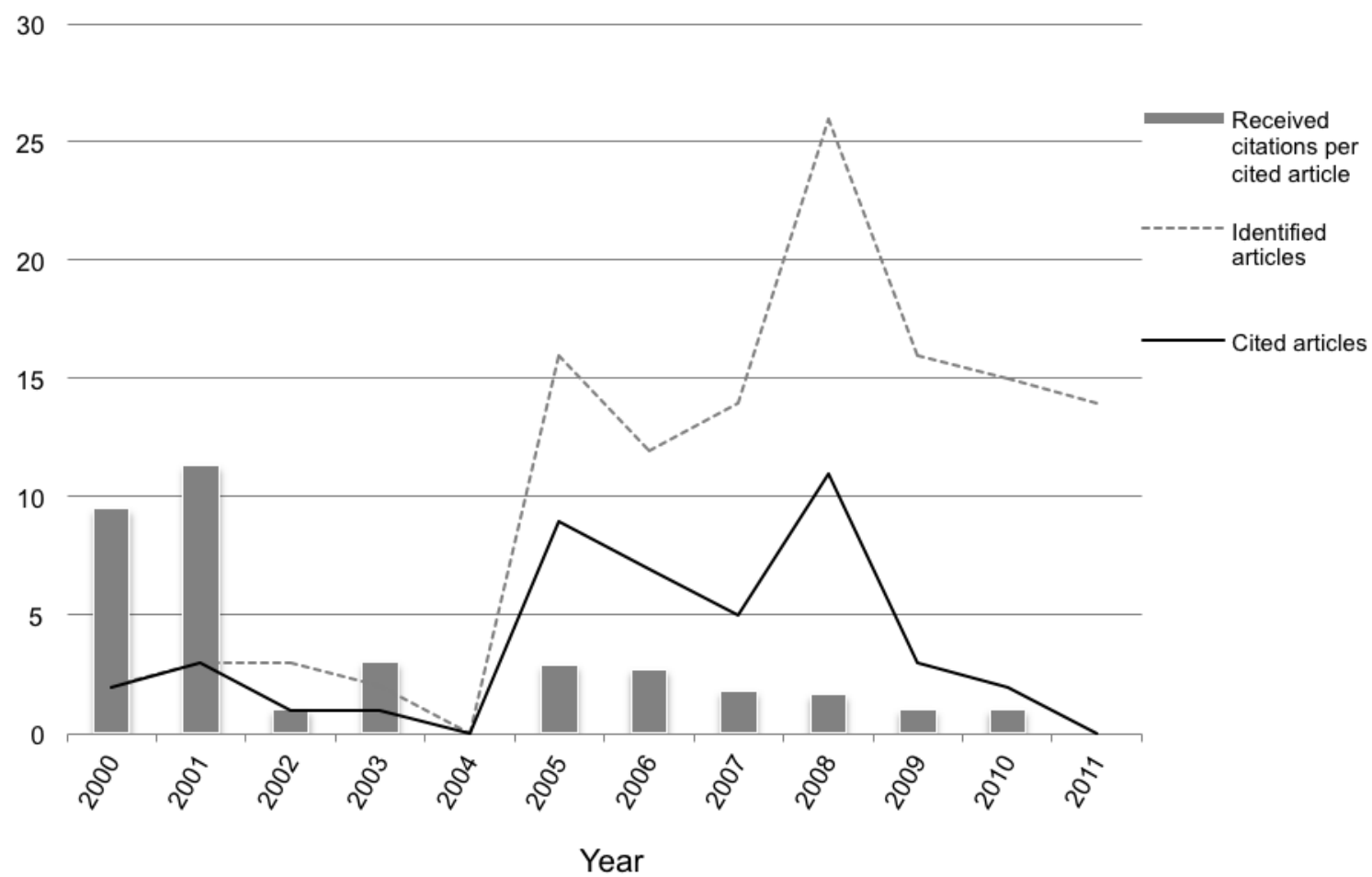


Figure 6: Further considered variables apart from effort.

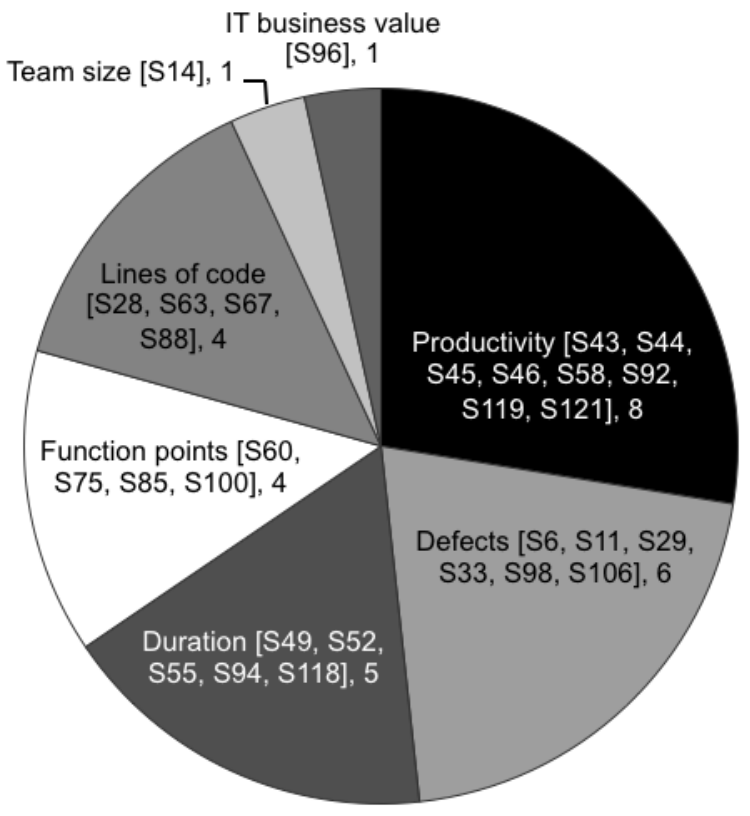


Figure 7: Dataset properties.

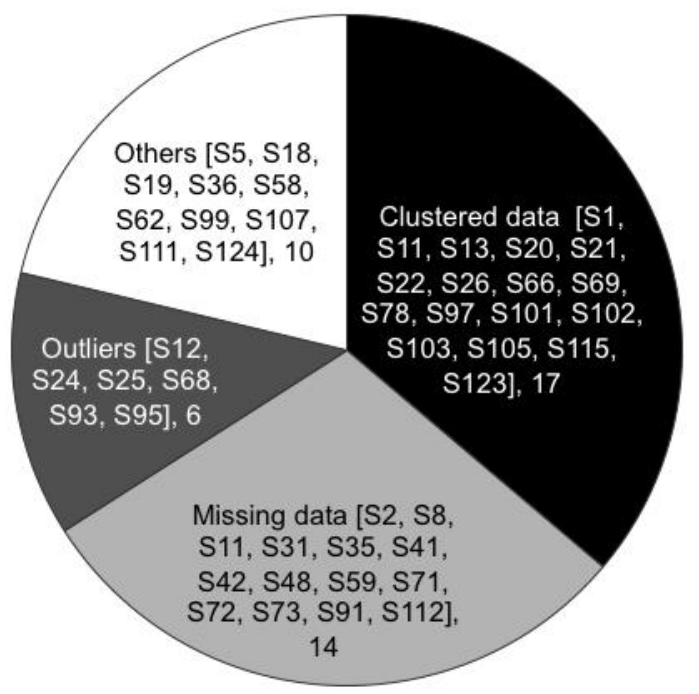


Figure 8: Evolution over time of the three most frequent topics.

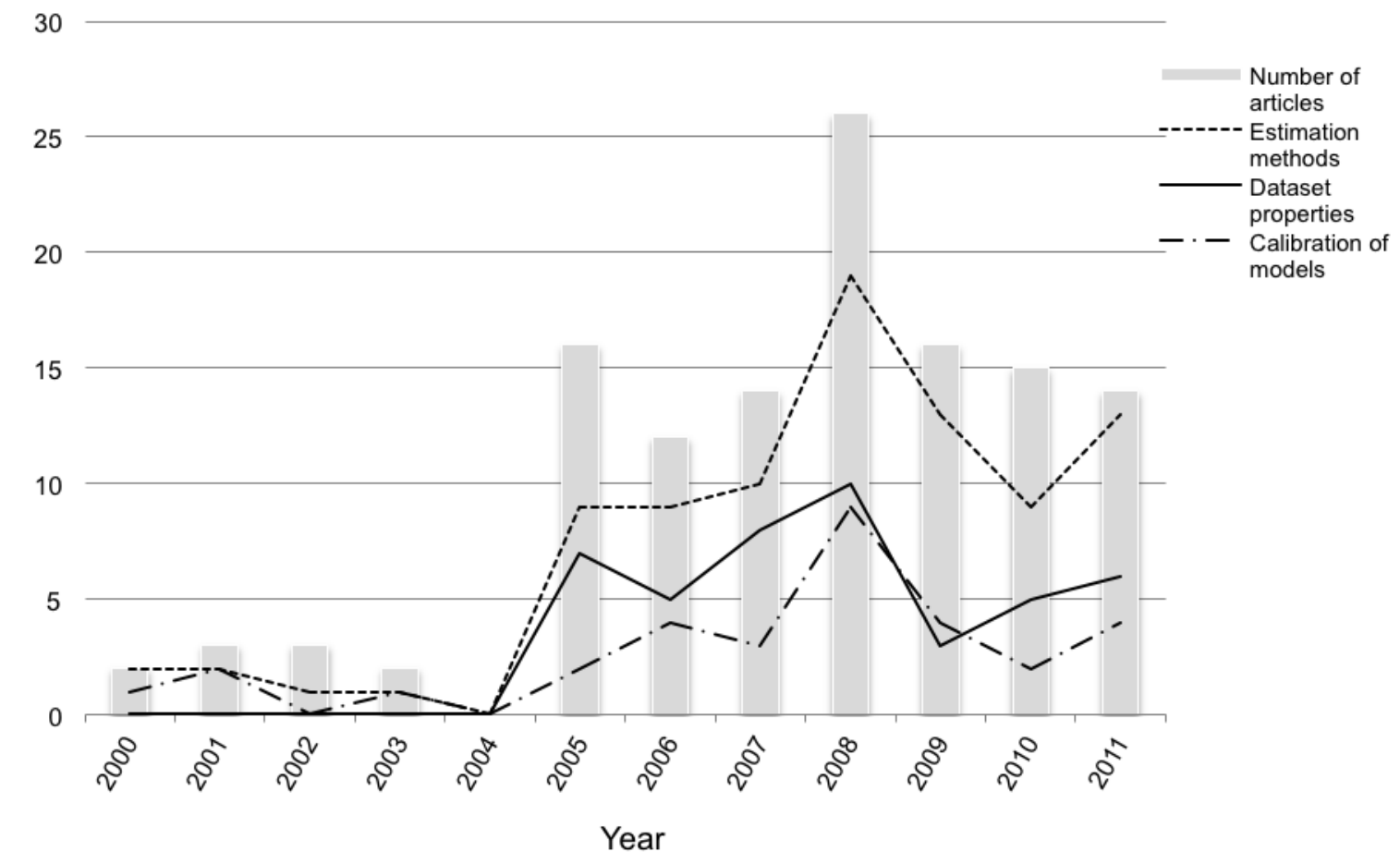


Figure 9: Evolution over time of the three most frequent estimation methods used.

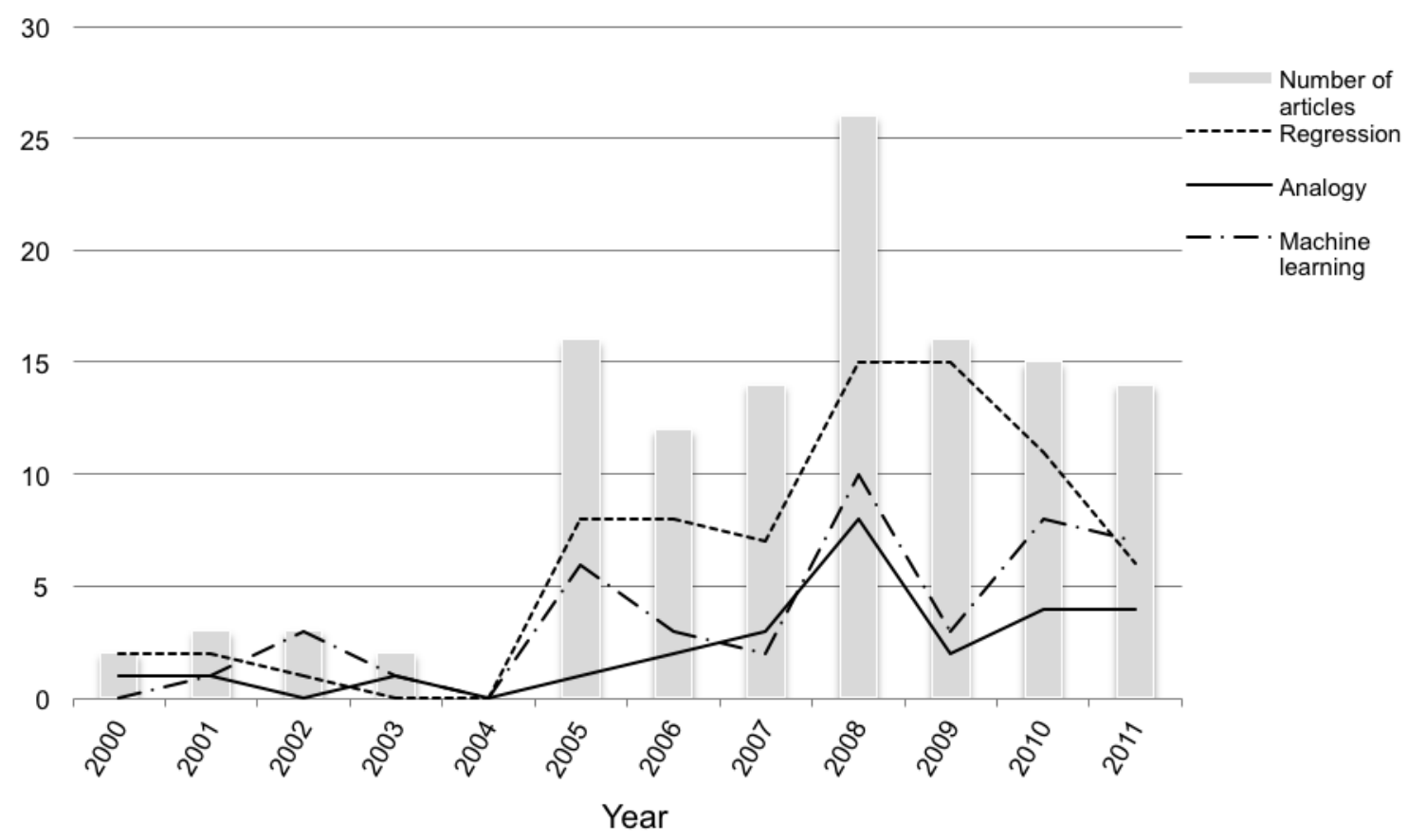


Figure 10: Evolution over time of the three most frequently used estimation methods in journals with ISBSG-based research papers.

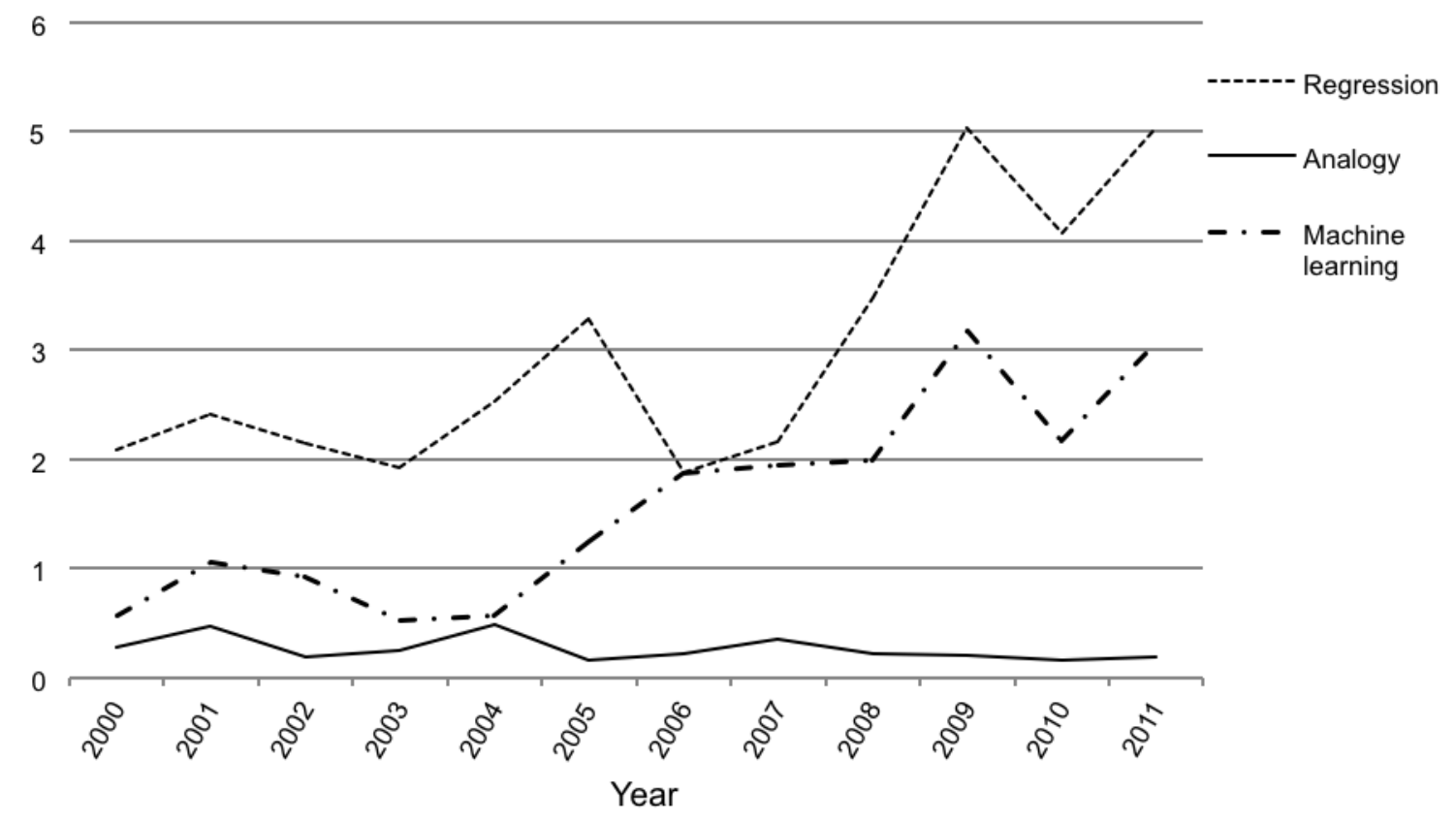


Figure 11: ISBSG releases and the evolution of the number of papers using a specific release of ISBSG.

(a): ISBSG releases over time.

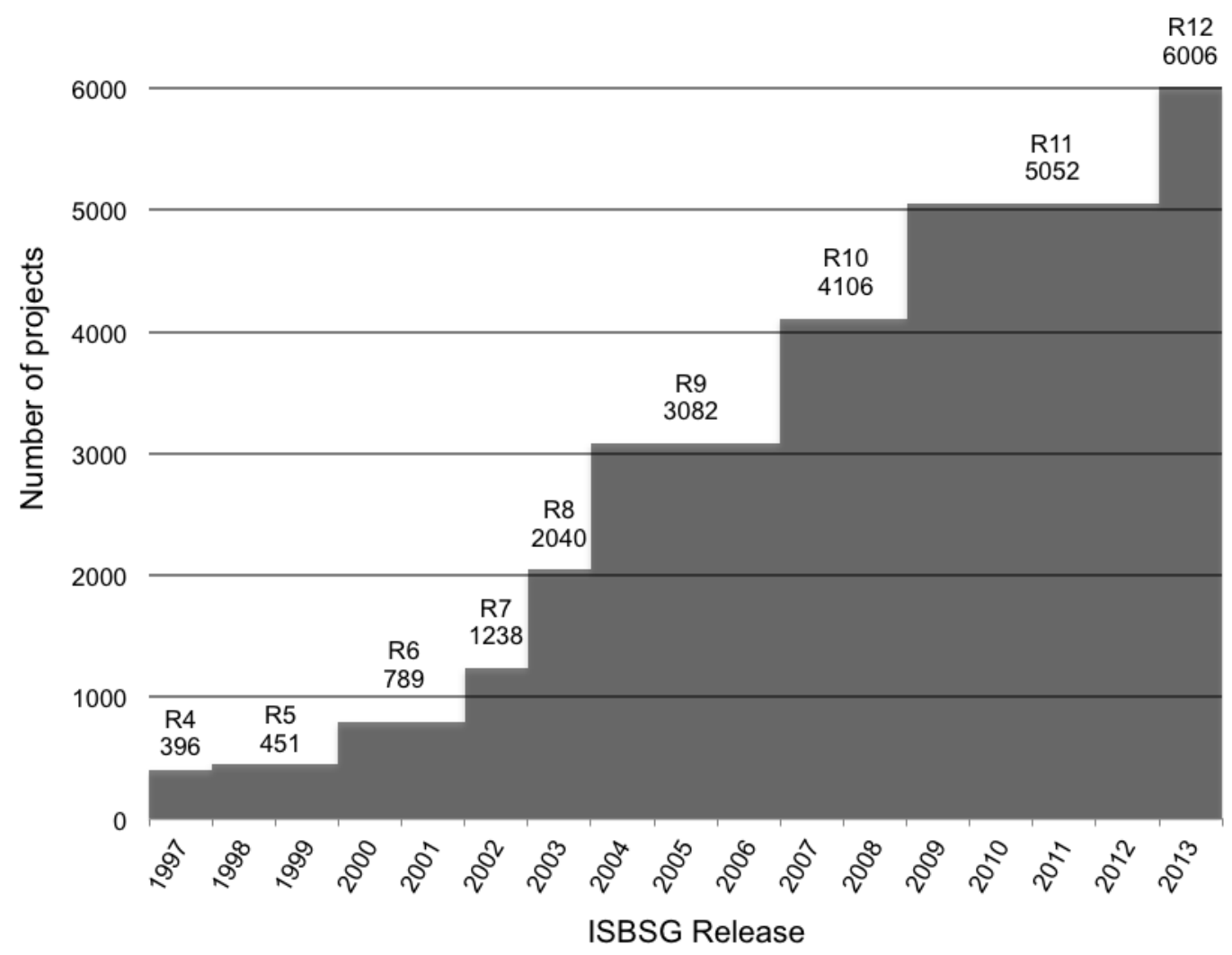

(b): Evolution of the number of papers using a specific release of ISBSG.

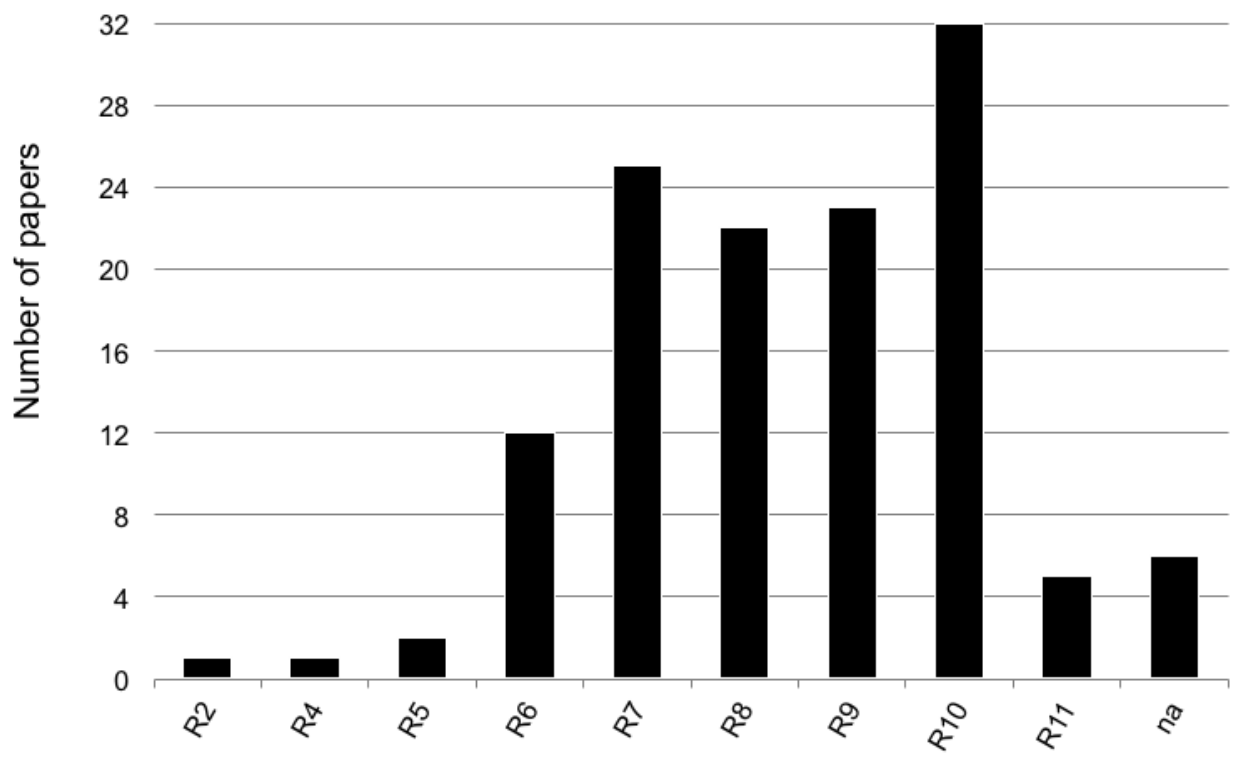

ISBSG Release 

Table 1: Data extraction form to collect information from each identified paper.

\begin{tabular}{ll}
\hline $\begin{array}{l}\text { Research } \\
\text { Question }\end{array}$ & \\
\hline RQ1 & - Only general information: Title, author(s), type, source, year, keywords, abstract. \\
RQ2 & - Received citations. \\
& - Number of received citations in Google Scholar*. \\
RQ3 & - Research topic. \\
& - Dependent variable. \\
RQ4 & - Estimation method. \\
RQ5 & - ISBSG release. \\
& - ISBSG used alone (Y/N). \\
& - Identification of complementary datasets (if any). \\
& - Justification for the use of complementary datasets. \\
& - Advantages and limitations of ISBSG dataset. \\
\hline
\end{tabular}

* Complementary information obtained from external sources. 
Table 2: Journal type publication sources of selected papers.

\begin{tabular}{|c|c|c|c|c|}
\hline Journal & Number & on $\%$ & $\begin{array}{l}\text { Cumulative } \\
\text { proportion } \%\end{array}$ & king \\
\hline Journal of Systems and Software & 11 & 17.7 & 17.7 & Q3 \\
\hline Information and Software Technology & 10 & 16.1 & 33.9 & Q2 \\
\hline Empirical Software Engineering & 9 & 14.5 & 48.4 & Q1 \\
\hline Software Quality Journal & 7 & 11.3 & 59.7 & Q4 \\
\hline $\begin{array}{l}\text { International Journal of Software Engineering and } \\
\text { Knowledge Engineering }\end{array}$ & 3 & 4.8 & 64.5 & Q4 \\
\hline $\begin{array}{l}\text { Journal of Software Maintenance and Evolution- } \\
\text { Research and Practice }\end{array}$ & 3 & 4.8 & 69.4 & Q3 \\
\hline Information \& Management & 2 & 3.2 & 72.6 & Q1* \\
\hline Journal of Computer Science and Technology & 2 & 3.2 & 75.8 & Q3 \\
\hline SIGSOFT Softw. Eng. Notes & 2 & 3.2 & 79.0 & \\
\hline Software Engineering, IEEE Transactions on & 2 & 3.2 & 82.3 & Q1 \\
\hline Software, IEEE & 2 & 3.2 & 85.5 & Q1 \\
\hline Software, IET & 2 & 3.2 & 88.7 & Q4 \\
\hline ACM Trans. Softw. Eng. Methodol. & 1 & 1.6 & 90.3 & Q2 \\
\hline Annals of Operations Research & 1 & 1.6 & 91.9 & Q3* \\
\hline Commun. ACM & 1 & 1.6 & 93.5 & Q1 \\
\hline Computing and Informatics & 1 & 1.6 & 95.2 & Q4* \\
\hline Expert Systems with Applications & 1 & 1.6 & 96.8 & Q1* \\
\hline Information Processing Letters & 1 & 1.6 & 98.4 & Q4* \\
\hline Science of Computer Programming & 1 & 1.6 & 100.0 & Q3 \\
\hline
\end{tabular}

* Quartile in category other than Computer Science, Software Engineering. 
Table 3: Conference type sources with two or more selected papers.

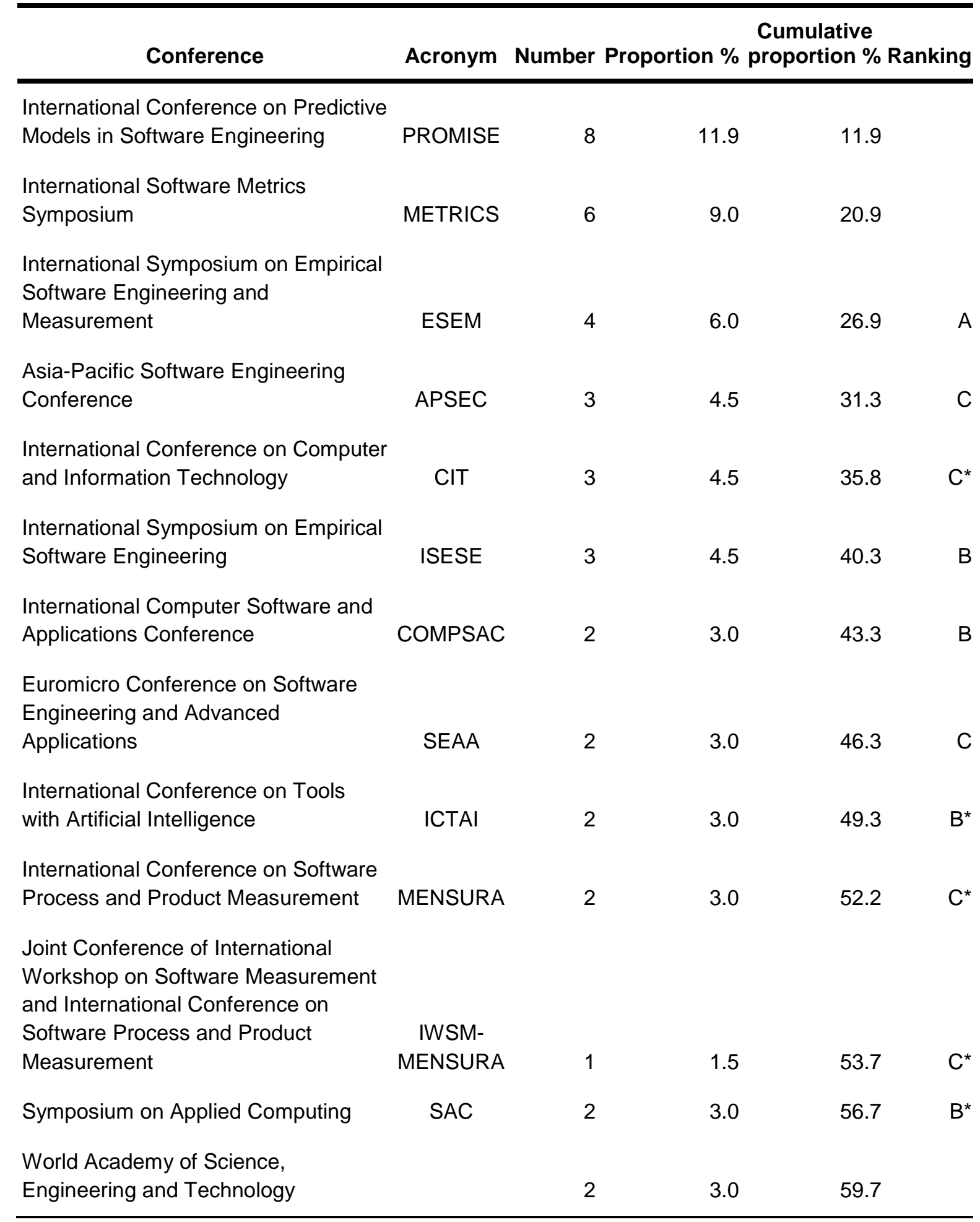

* Tier ranking in field other than Computer Software. 
Table 4: Top-cited identified papers with at least 6 citations.

\begin{tabular}{llccc}
\hline Ref. & \multicolumn{1}{c}{ Source } & Year & $\begin{array}{c}\text { Number of received } \\
\text { citations }\end{array}$ & $\begin{array}{c}\text { Received citations } \\
\text { Google Scholar }\end{array}$ \\
\hline$[$ S125] & METRICS & 2001 & 15 & 145 \\
{$[$ S40] } & METRICS & 2001 & 14 & 91 \\
& $\begin{array}{l}\text { Information and } \\
\text { Software }\end{array}$ & & 13 & 126 \\
[S4] & $\begin{array}{l}\text { Technology } \\
\text { Information and }\end{array}$ & 2000 & & 78 \\
{$[$ S114] } & $\begin{array}{l}\text { Technology } \\
\text { Information and }\end{array}$ & 2005 & 10 & \\
\hline S23] & Teftware & & 6 & 57 \\
\hline
\end{tabular}


Table 5: Citations received by identified papers in dataset journals over time.

\begin{tabular}{|c|c|c|c|c|c|c|c|}
\hline Year & $\begin{array}{l}\text { Identified } \\
\text { articles }\end{array}$ & $\begin{array}{c}\text { Cited } \\
\text { articles }\end{array}$ & $\begin{array}{l}\text { Number } \\
\text { of } \\
\text { received } \\
\text { citations }\end{array}$ & $\begin{array}{l}\text { Identified } \\
\text { cited } \\
\text { articles } \\
\%\end{array}$ & $\begin{array}{l}\text { Received } \\
\text { citations } \\
\text { per } \\
\text { identified } \\
\text { article }\end{array}$ & $\begin{array}{l}\text { Received } \\
\text { citations } \\
\text { per cited } \\
\text { article }\end{array}$ & $\begin{array}{l}\text { Received } \\
\text { citations } \\
\text { on Google } \\
\text { Scholar }\end{array}$ \\
\hline 2000 & 2 & 2 & 19 & 100.0 & 9.5 & 9.5 & 183 \\
\hline 2001 & 3 & 3 & 34 & 100.0 & 11.3 & 11.3 & 275 \\
\hline 2002 & 3 & 1 & 1 & 33.3 & 0.3 & 1.0 & 38 \\
\hline 2003 & 2 & 1 & 3 & 50.0 & 1.5 & 3.0 & 37 \\
\hline 2004 & 0 & 0 & 0 & 0.0 & 0.0 & 0.0 & - \\
\hline 2005 & 16 & 9 & 26 & 56.2 & 1.6 & 2.9 & 222 \\
\hline 2006 & 12 & 7 & 19 & 58.3 & 1.6 & 2.7 & 175 \\
\hline 2007 & 14 & 5 & 9 & 35.7 & 0.6 & 1.8 & 150 \\
\hline 2008 & 26 & 11 & 18 & 42.3 & 0.7 & 1.6 & 176 \\
\hline 2009 & 16 & 3 & 3 & 18.7 & 0.2 & 1.0 & 55 \\
\hline 2010 & 15 & 2 & 2 & 13.3 & 0.1 & 1.0 & 26 \\
\hline 2011 & 14 & 0 & 0 & 0.0 & 0.0 & 0.0 & - \\
\hline Total & 123 & 44 & 134 & 35.8 & 1.1 & 3.0 & 1337 \\
\hline
\end{tabular}


Table 6: Number and proportion of papers for each research topic.

\begin{tabular}{lrr}
\hline \multicolumn{1}{c}{ Research topic } & $\begin{array}{c}\text { Number } \\
\text { of papers }\end{array}$ & $\begin{array}{c}\text { Proportion } \\
\%\end{array}$ \\
\hline Estimation methods & 91 & 70.5 \\
Dataset properties & 47 & 36.4 \\
Calibration of models & 32 & 24.8 \\
Production functions & 18 & 13.9 \\
Size measures & 15 & 11.6 \\
Organizational issues & 11 & 8.5 \\
Measures of estimation performance & 6 & 4.6 \\
Phase effort estimation & 4 & 3.1 \\
Effort uncertainty assessments & 2 & 1.5 \\
Other topics & 21 & 16.3 \\
\hline
\end{tabular}


Table 7: Number and proportion of papers for each estimation method.

\begin{tabular}{lcr}
\hline \multicolumn{1}{c}{ Method } & $\begin{array}{c}\text { Number of } \\
\text { papers }\end{array}$ & Proportion \% \\
\hline Regression & 79 & 61.2 \\
Machine Learning & 46 & 35.7 \\
Estimation by Analogy & 29 & 22.5 \\
Combination of Estimates & 17 & 13.2 \\
Function Point & 8 & 6.2 \\
Fuzzy & 8 & 6.2 \\
Simulation & 3 & 2.3 \\
Not relevant & 7 & 5.4 \\
Others & 18 & 13.9 \\
\hline
\end{tabular}


Table 8: Machine Learning estimation methods.

\begin{tabular}{lcc}
\hline \multicolumn{1}{c}{ Machine Learning method } & $\begin{array}{c}\text { Number } \\
\text { of papers }\end{array}$ & $\begin{array}{c}\text { Proportion } \\
\%\end{array}$ \\
\hline Neural Networks (NN) & 18 & 13.9 \\
Model tree & 10 & 7.7 \\
Classification and & 9 & 7.0 \\
Regression Trees (CART) & 7 & \\
Genetic Algorithms (GA) & 6 & 5.4 \\
Support Vector Machines (SVM) & 5 & 4.6 \\
Bayesian Networks (BN) & 3 & 3.9 \\
Association Rules (AR) & 16 & 2.3 \\
Others & 5.4 \\
\hline
\end{tabular}


Table 9: Other datasets used apart from ISBSG.

\begin{tabular}{lcccc}
\hline \multicolumn{1}{c}{ Dataset acronym } & Source & $\begin{array}{r}\text { Number of } \\
\text { papers }\end{array}$ & $\begin{array}{c}\text { Public / } \\
\text { Limited }\end{array}$ & PROMISE \\
\hline Desharnais & {$[24]$} & 20 & $\mathrm{P}$ & $\mathrm{Y}$ \\
Cocomo81 & {$[25]$} & 14 & $\mathrm{P}$ & $\mathrm{Y}$ \\
STTF = Bank63 & {$[17]$} & 14 & $\mathrm{P}$ & $\mathrm{Y}$ \\
Kem87 & {$[26]$} & 13 & $\mathrm{P}$ & $\mathrm{Y}$ \\
Albrecht (\& Gaffney) & {$[27]$} & 12 & $\mathrm{P}$ & $\mathrm{N}$ \\
Nasa93 = Cocnasa & {$[28]$} & 8 & $\mathrm{P}$ & $\mathrm{Y}$ \\
USP05 & {$[$ S5] } & 7 & $\mathrm{P}$ & $\mathrm{N}$ \\
Mendes03 & {$[29]$} & 5 & $\mathrm{P}$ & $\mathrm{N}$ \\
Finnish = Experience & {$[30]$} & 4 & $\mathrm{~L}$ & $\mathrm{~N}$ \\
Sdr & {$[28,31]$} & 4 & $\mathrm{P}$ & $\mathrm{Y}$ \\
Abran \& Robilard & {$[32]$} & 3 & $\mathrm{P}$ & $\mathrm{N}$ \\
CSBSG & {$[33]$} & 2 & $\mathrm{~L}$ & $\mathrm{~N}$ \\
China & {$[28]$} & 2 & $\mathrm{P}$ & $\mathrm{Y}$ \\
\hline
\end{tabular}




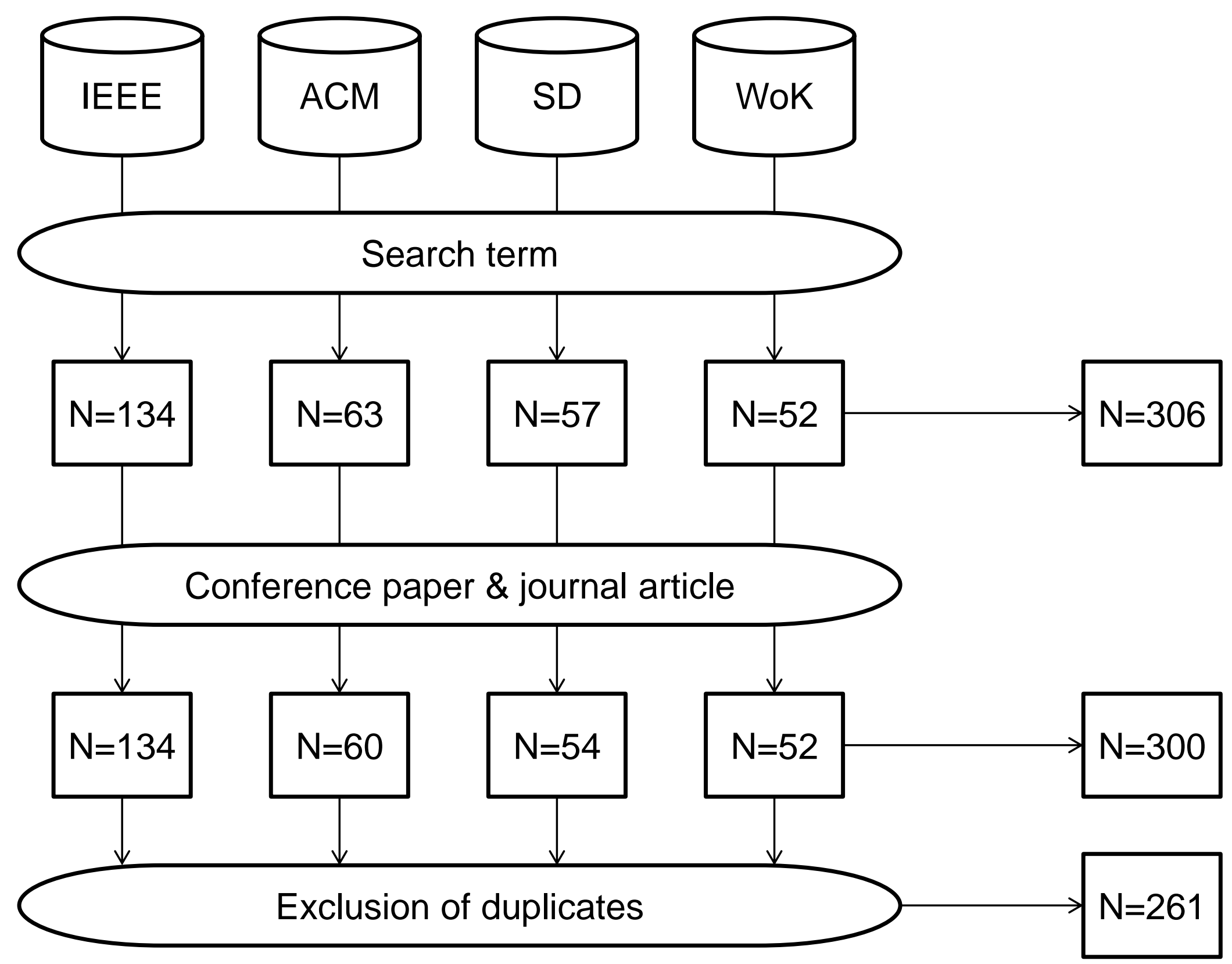




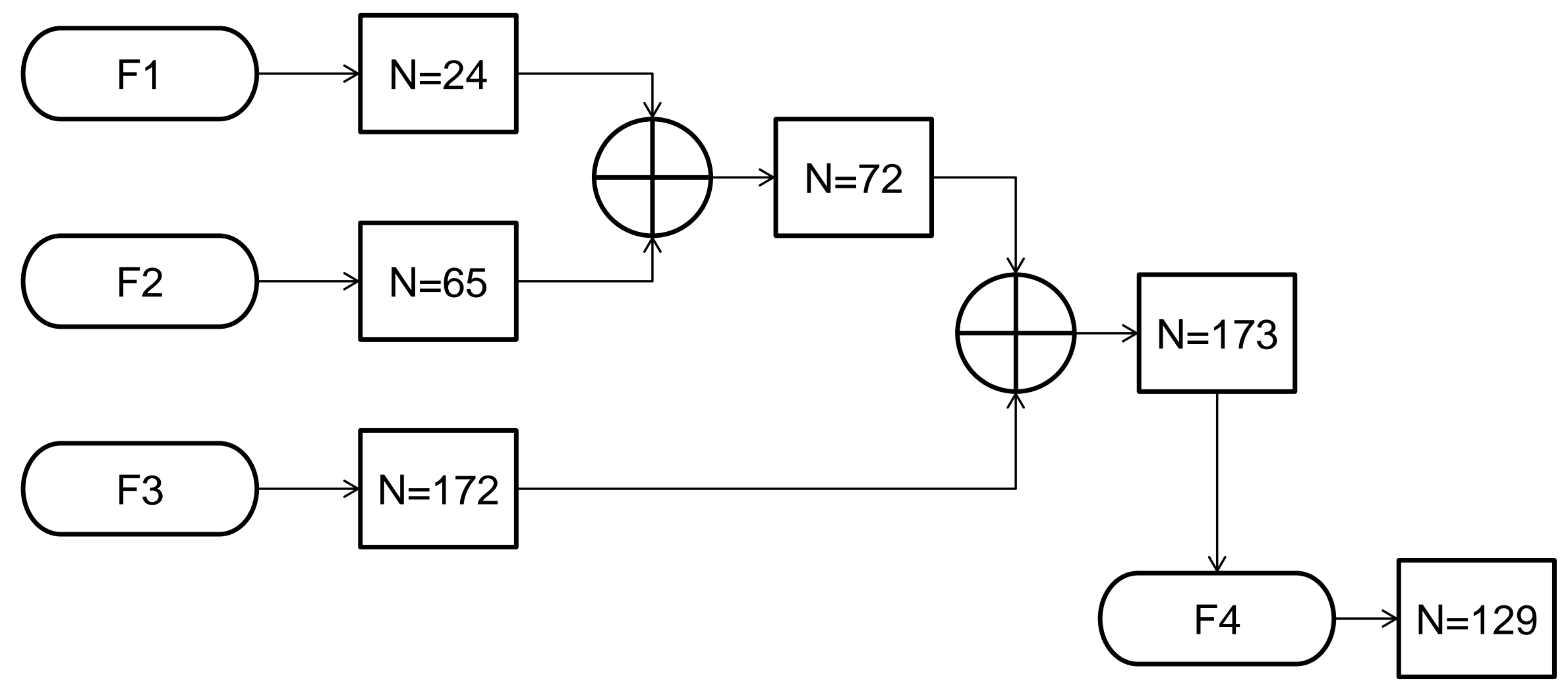


Figure xIs

$\begin{array}{rrr}\text { Year } & \text { In journals } & \text { In conferen } \\ 2000 & 2 & \\ 2001 & 1 & 2 \\ 2002 & 1 & 2 \\ 2003 & 1 & 1 \\ 2004 & & \\ 2005 & 9 & 7 \\ 2006 & 5 & 7 \\ 2007 & 6 & 8 \\ 2008 & 15 & 11 \\ 2009 & 5 & 11 \\ 2010 & 5 & 10 \\ 2011 & 6 & 8 \\ & & \\ 2012 & & \end{array}$

2012 